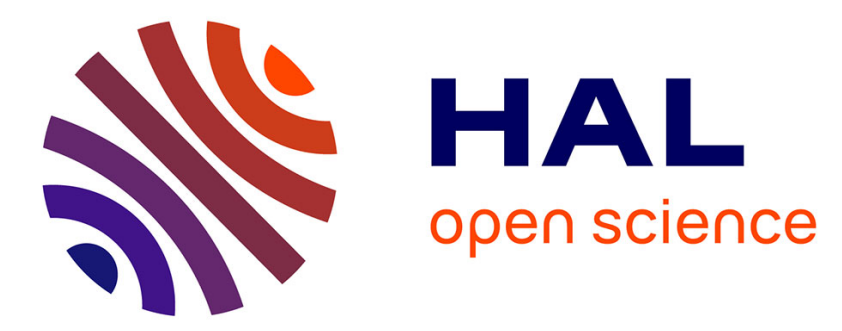

\title{
A Fresh Look at the Bayesian Bounds of the Weiss-Weinstein Family
}

Alexandre Renaux, Philippe Forster, Pascal Larzabal, Christ Richmond, Arye Nehorai

\section{- To cite this version:}

Alexandre Renaux, Philippe Forster, Pascal Larzabal, Christ Richmond, Arye Nehorai. A Fresh Look at the Bayesian Bounds of the Weiss-Weinstein Family. IEEE Transactions on Signal Processing, 2008, 56 (11), pp.5334-5352. inria-00444764

\section{HAL Id: inria-00444764 https://hal.inria.fr/inria-00444764}

Submitted on 7 Jan 2010

HAL is a multi-disciplinary open access archive for the deposit and dissemination of scientific research documents, whether they are published or not. The documents may come from teaching and research institutions in France or abroad, or from public or private research centers.
L'archive ouverte pluridisciplinaire HAL, est destinée au dépôt et à la diffusion de documents scientifiques de niveau recherche, publiés ou non, émanant des établissements d'enseignement et de recherche français ou étrangers, des laboratoires publics ou privés. 


\title{
A Fresh Look at the Bayesian Bounds of the Weiss-Weinstein Family
}

\author{
Alexandre Renaux, Member, IEEE, Philippe Forster, Member, IEEE, Pascal Larzabal, Member, IEEE, \\ Christ D. Richmond, Senior Member, IEEE, and Arye Nehorai, Fellow, IEEE
}

\begin{abstract}
Minimal bounds on the mean square error (MSE) are generally used in order to predict the best achievable performance of an estimator for a given observation model. In this paper, we are interested in the Bayesian bound of the Weiss-Weinstein family. Among this family, we have Bayesian Cramér-Rao bound, the Bobrovsky-MayerWolf-Zakaï bound, the Bayesian Bhattacharyya bound, the Bobrovsky-Zakaï bound, the Reuven-Messer bound, and the Weiss-Weinstein bound. We present a unification of all these minimal bounds based on a rewriting of the minimum mean square error estimator (MMSEE) and on a constrained optimization problem. With this approach, we obtain a useful theoretical framework to derive new Bayesian bounds. For that purpose, we propose two bounds. First, we propose a generalization of the Bayesian Bhattacharyya bound extending the works of Bobrovsky, Mayer-Wolf, and Zakaï. Second, we propose a bound based on the Bayesian Bhattacharyya bound and on the Reuven-Messer bound, representing a generalization of these bounds. The proposed bound is the Bayesian extension of the deterministic Abel bound and is found to be tighter than the Bayesian Bhattacharyya bound, the Reuven-Messer bound, the Bobrovsky-Zakaï bound, and the Bayesian Cramér-Rao bound. We propose some closed-form expressions of these bounds for a general Gaussian observation model with parameterized mean. In order to illustrate our results, we present simulation results in the context of a spectral analysis problem.
\end{abstract}

Index Terms-Bayesian bounds on the MSE, Weiss-Weinstein family.

Manuscript received April 24, 2007; revised April 18, 2008 First published June 13, 2008; current version published October 15, 2008. The associate editor coordinating the review of this manuscript and approving it for publication was Prof. Philippe Loubaton. The work of A. Renaux and A. Nehorai was supported in part by the Department of Defense under the Air Force Office of Scientific Research MURI Grant FA9550-05-1-0443, AFOSR Grant FA9550-05-1-0018, and by the National Science Foundation by Grants CCR-0330342 and CCF0630734. The material in this paper was presented in part at the IEEE Workshop on Statistical Signal Processing, Bordeaux, France, July 2005 and at the IEEE International Conference on Acoustic, Speech and Signal Processing, Toulouse, France, May 2006.

A. Renaux is with the Laboratoire des Signaux et Systèmes, Laboratory of Signals and Systems, University Paris-Sud 11, 91192 Gif-sur-Yvette cedex, France (e-mail: renaux@1ss.supelec.fr).

P. Forster is with the Universite de Nanterre, IUT de Ville d'Avray, France. $\mathrm{He}$ is also with the SATIE Laboratory (École Normale Supérieure de Cachan), 94235 Cachan, France (e-mail: pforster@u-paris 10.fr).

P. Larzabal is with the University Paris-Sud 11,91192 Gif-sur-Yvette cedex, France. He is also with the SATIE Laboratory (École Normale Supérieure de Cachan), 94235 Cachan, France (e-mail: larzabal@ satie.ens-cachan.fr).

C. D. Richmond is with the Advanced Sensor Techniques Group, Lincoln Laboratory, Massachusetts Institute of Technology, Lexington, MA 02420 USA (e-mail: christ@1l.mit.edu).

A. Nehorai is with Washington University, St. Louis, MO 63130 USA (e-mail: nehorai@ese.wustl.edu).

Color versions of one or more of the figures in this paper are available online at http://ieeexplore.ieee.org.

Digital Object Identifier 10.1109/TSP.2008.927075

\section{NOTATIONS}

The notational convention adopted in this paper is as follows: italic indicates a scalar quantity, as in $A$; lowercase boldface indicates a vector quantity, as in a; uppercase boldface indicates a matrix quantity, as in $\mathbf{A} . \operatorname{Re}\{A\}$ is the real part of $A$ and $\operatorname{Im}\{A\}$ is the imaginary part of $A$. The complex conjugation of a quantity is indicated by a superscript * as in $A^{*}$. The matrix transpose is indicated by a superscript ${ }^{T}$ as in $\mathbf{A}^{T}$, and the complex conjugate plus matrix transpose is indicated by a superscript ${ }^{H}$ as in $\mathbf{A}^{H}=\left(\mathbf{A}^{T}\right)^{*}$. The $n$-th row and $m$-th column element of the matrix $\mathbf{A}$ is denoted by $\{\mathbf{A}\}_{n, m}$. $\mathbf{I}_{N}$ denotes the identity matrix of size $N \times N$. $\mathbf{0}_{N \times M}$ is a $N \times M$ matrix of zeros. $\|\cdot\|$ denotes the norm. $|\cdot|$ denotes the modulus. abs $(\cdot)$ denotes the absolute value. $\delta(\cdot)$ denotes the Dirac delta function. $\mathbb{E}[\cdot]$ denotes the expectation operator with respect to a density probability function explicitly given by a subscript. $\Omega$ is the observation space and $\Theta$ is the parameter space.

\section{INTRODUCTION}

M INIMAL bounds on the mean square error (MSE) provide the ultimate performance that an estimator can expect to achieve for a given observation model. Consequently, they are used as a benchmark in order to evaluate the performance of an estimator and to determine if an improvement is possible. The Cramér-Rao bound [3]-[8] has been the most widely used by the signal processing community and is still under investigation from a theoretical point of view (particularly throughout the differential variety in the Riemannian geometry framework [9]-[14]) as from a practical point of view (see, e.g., [15]-[19]). But the Cramér-Rao bound suffers from some drawbacks when the scenario becomes critical. Indeed, in a nonlinear estimation problem, when the parameters have finite support, there are three distinct MSE areas for an estimator [20, p. 273], [21]. For a large number of observations or for a high signal-to-noise ratio (SNR), the estimator MSE is small and the area is called an asymptotic area. When the scenario becomes critical, i.e., when the number of observations or the SNR decreases, the estimator MSE increases dramatically due to the outlier effect, and the area is called threshold area. Finally, when the number of observations or the SNR is low, the estimator criterion is hugely corrupted by the noise and becomes a quasi-uniform random variable on the parameter support. Since in this last area the observations bring almost no information, it is called no information area. The Cramér-Rao bound is used only in the asymptotic area and is not able to handle the threshold phenomena (i.e., when the performance breaks down). 
To fill this lack, a plethora of other minimal bounds tighter than the Cramér-Rao bound has been proposed and studied. All these bounds have been derived by way of several inequalities, such as the Cauchy-Schwartz inequality, the Kotelnikov inequality, the Hölder inequality, the Ibragimov-Hasminskii inequality, the Bhattacharyya inequality, and the Kiefer inequality. Note that due to this diversity, it is sometimes difficult to fully understand the underlying concept and the difference between all these bounds; consequently it is difficult to apply these bounds to a specific estimation problem.

Minimal bounds on the MSE can be divided into two categories: the deterministic bounds for situations in which the true vector of the parameters $\boldsymbol{\theta}_{0}$ is assumed to be deterministic and the Bayesian bounds for situations in which the vector of parameters $\boldsymbol{\theta}$ is assumed to be random with an a priori probability density function $p(\boldsymbol{\theta})$. Among the deterministic bounds, we have the well-known Cramér-Rao bound; the Bhattacharyya bound [22], [23]; the Chapman-Robbins bound [24]-[26], the Barankin bound [27], [28], the Abel bound [29]-[31]; and the Quinlan-Chaumette-Larzabal bound [32]. Bayesian bounds can be subdivided into two categories: the Ziv-Zakaï family, derived from a binary hypothesis testing problem (and more generally from an $M$-ary hypothesis testing problem), and the WeissWeinstein family, derived (as the deterministic bounds) from a covariance inequality principle. The Ziv-Zakaï family contains the Ziv-Zakaï bound [33], the Bellini-Tartara bound [34], the Chazan-Zakaï-Ziv bound [35], the Weinstein bound [36], the Bell-Steinberg-Ephraim-VanTrees bound [37], and the Bell bound [38]. The Weiss-Weinstein family contains the Bayesian Cramér-Rao bound [20, pp. 72 and 84], the Bobrovsky-MayerWolf-Zakaï bound [39], the Bayesian Bhattacharyya bound [20, p. 149], the Bobrovsky-Zakaï bound [40], the Reuven-Messer bound [41], and the Weiss-Weinstein bound [42]. A nice tutorial about both families can be found in the recent book of Van Trees and Bell [43].

The deterministic bounds are used as a lower bound of the local MSE in $\boldsymbol{\theta}_{0}$; i.e.,

$$
\mathbf{M S E}_{L}\left(\boldsymbol{\theta}_{0}\right)=\int_{\Omega}\left(\hat{\boldsymbol{\theta}}(\mathbf{y})-\boldsymbol{\theta}_{0}\right)\left(\hat{\boldsymbol{\theta}}(\mathbf{y})-\boldsymbol{\theta}_{0}\right)^{T} p\left(\mathbf{y} \mid \boldsymbol{\theta}_{0}\right) d \mathbf{y}
$$

where $\mathbf{y} \in \Omega$ is a complex observation vector, $p\left(\mathbf{y} \mid \boldsymbol{\theta}_{0}\right)$ is the likelihood of the observations parameterized by the true parameter value $\boldsymbol{\theta}_{0}$, and $\hat{\boldsymbol{\theta}}(\mathbf{y})$ is an estimator of $\boldsymbol{\theta}_{0}$.

On the other hand, Bayesian bounds are used as a lower bound of the global MSE; i.e.,

$$
\mathbf{M S E}_{G}=\int_{\Theta} \int_{\Omega}(\hat{\boldsymbol{\theta}}(\mathbf{y})-\boldsymbol{\theta})(\hat{\boldsymbol{\theta}}(\mathbf{y})-\boldsymbol{\theta})^{T} p(\mathbf{y}, \boldsymbol{\theta}) d \mathbf{y} d \boldsymbol{\theta}
$$

where $\boldsymbol{\theta} \in \boldsymbol{\Theta}$ is the random parameter vector with an a priori probability density function $p(\boldsymbol{\theta})=(p(\mathbf{y}, \boldsymbol{\theta}) / p(\mathbf{y} \mid \boldsymbol{\theta}))$ and $p(\mathbf{y}, \boldsymbol{\theta})$ is the joint probability function of the observations and of the parameters.

In the deterministic context, minimal bounds - in particular the Chapman-Robbins bound and the Barankin bound-are generally used in order to predict the aforementioned threshold effect which cannot be handled by the Cramér-Rao bound. The Chapman-Robbins bound and the Barankin bound have already been successfully applied to several estimation problems [28], [31], [44]-[55]. The use of the Abel bound, which can also handle the threshold phenomena, is still marginal [56].

Contrary to the deterministic bounds, the Bayesian bounds take into account the parameter support throughout the a priori probability density function $p(\boldsymbol{\theta})$, and they give the ultimate performances of an estimator on the three aforementioned areas of the global MSE. These bounds give the performance of the Bayesian estimator, such as the maximum a posteriori (MAP) estimator or the minimum mean square error estimator (MMSEE), and can be used in order to know the global performance of the deterministic estimators such as the maximum likelihood estimator (MLE), since

$$
\mathbf{M S E}_{G}=\int_{\Theta} \mathbf{M S E}_{L}(\boldsymbol{\theta}) p(\boldsymbol{\theta}) d \boldsymbol{\theta} .
$$

The reader is referred to $\mathrm{Xu}$ et al. [57]-[59], where the MLE performances are analyzed in the context of an underwater acoustic problem by way of the Ziv-Zakaï and of the Weiss-Weinstein bounds. The Ziv-Zakaï family bounds have been applied in other signal processing areas: time-delay estimation [60]; direction-of-arrival estimation [38], [61], [62]; and digital communication [63]. On the other hand, the Weiss-Weinstein bound has been less investigated: the aforementioned $\mathrm{Xu}$ et al. works and in the framework of digital communication [64].

This article presents a new unified approach for the establishment of the Weiss-Weinstein family bounds. Note that the unification of the deterministic bounds has already been proposed by [65] and [66] based on a constrained optimization problem. A unification has been proposed by Bell et al. in [37] and [38] for the Ziv-Zakaï family.

Concerning the Weiss-Weinstein family unification, a first approach has been given by Weiss and Weinstein in [67]. This approach is based on the following inequality proved by the authors:

$$
\operatorname{MSE}_{G} \geq \frac{\mathbb{E}_{\mathbf{y}, \theta}^{2}[\theta \psi(\mathbf{y}, \theta)]}{\mathbb{E}_{\mathbf{y}, \theta}\left[\psi^{2}(\mathbf{y}, \theta)\right]}
$$

where the function $\psi(\mathbf{y}, \theta)$ must satisfied

$$
\int_{\Theta} \psi(\mathbf{y}, \theta) p(\mathbf{y}, \theta) d \theta=0 .
$$

Weiss and Weinstein gave several functions $\psi(\mathbf{y}, \theta)$ satisfying (5) for which they again obtain the Bayesian Cramér-Rao bound, the Bayesian Bhattacharyya bound, the Bobrovsky-Zakaï bound, and the Weiss-Weinstein bound. Moreover, a function $\psi(\mathbf{y}, \theta)$ satisfying (5) leading to the Bobrovsky-MayerWolf-Zakaï bound is given in [38]. Unfortunately, there are no general rules to find $\psi(\mathbf{y}, \theta)$. In this contribution, the Weiss-Weinstein family unification is based on the best Bayesian bound, i.e., the MSE of the MMSEE. By rewriting the MMSEE and by using a constrained optimization problem similar to one derived for the unification of deterministic bounds [65], [66], we unify the Bayesian Cramér-Rao bound, the Bobrovsky-MayerWolf-Zakaï bound, the Bayesian Bhattacharyya bound, the Bobrovsky-Zakai 
bound, the Reuven-Messer bound (for which no function $\psi(\mathbf{y}, \theta)$ is proposed in the Weiss-Weinstein approach), and the Weiss-Weinstein bound. This approach brings a useful theoretical framework to derive new Bayesian bounds.

For that purpose, we propose two bounds. First, we propose a generalization of the Bayesian Bhattacharyya bound extending the works of Bobrovsky, Mayer-Wolf, and Zakaï. Second, we propose a bound based on the Bayesian Bhattacharyya bound and on the Reuven-Messer bound, one that represents a generalization of these bounds. This bound is found to be tighter than the Bayesian Bhattacharyya bound, the Reuven-Messer bound, the Bobrovsky-Zakaï bound, and the Bayesian Cramér-Rao bound. In order to illustrate our results, we propose some closed-form expressions of the minimal bounds for a Gaussian observation model with parameterized mean widely used in signal processing, and we apply it to a spectral analysis problem for which we present simulation results.

\section{MMSE REFORMULATION}

In this section, we start by reformulating the MMSEE as a constrained optimization problem. Then, we rewrite the underlying constraint under three different forms that will be of interest for our proposed unification.

In the Bayesian framework, the minimal global MSE and consequently the best Bayesian bound is the MSE of the MMSEE: $\hat{\theta}(\mathbf{y})=\int_{\Theta} \theta p(\theta \mid \mathbf{y}) d \theta$, where $p(\theta \mid \mathbf{y})$ is the a posteriori probability density function of the parameter. Unfortunately, it is generally impossible to obtain a closed-form expression of this MSE. The MMSEE is the solution of the following problem:

$$
\min _{\hat{\theta}(\mathbf{y})} \int_{\Theta} \int_{\Omega}(\hat{\theta}(\mathbf{y})-\theta)^{2} p(\mathbf{y}, \theta) d \mathbf{y} d \theta .
$$

Let $\mathcal{L}_{p}^{2}$ be the set of function $v(\mathbf{y}, \theta)$ such that $\int_{\Theta} \int_{\Omega} v^{2}(\mathbf{y}, \theta) p(\mathbf{y}, \theta) d \mathbf{y} d \theta$ is defined. Let $\mathcal{C}_{1} \subset \mathcal{L}_{p}^{2}$ be the subset of function satisfying

$$
v(\mathbf{y}, \theta)=z(\mathbf{y})-\theta
$$

where $z(\mathbf{y})$ is a function only of $\mathbf{y}$.
Consequently, the MMSEE (6) is the solution of the following constrained optimization problem:

$$
\left\{\begin{array}{l}
\min _{v} \iint_{\Theta} v^{2}(\mathbf{y}, \theta) p(\mathbf{y}, \theta) d \mathbf{y} d \theta \\
\text { subject to } v(\mathbf{y}, \theta) \in \mathcal{C}_{1}
\end{array} .\right.
$$

Let $\mathcal{F}$ be the set of functions $f(\mathbf{y}, \theta)$ such that $\forall v(\mathbf{y}, \theta) \in \mathcal{L}_{p}^{2}$

$$
\left\{\begin{array}{l}
\lim _{\theta \rightarrow \pm \infty} v(\mathbf{y}, \theta) f(\mathbf{y}, \theta)=0 \\
\lim _{\theta \rightarrow \pm \infty} \frac{\partial f(\mathbf{y}, \theta)}{\partial \theta}=0
\end{array}\right.
$$

Let us now introduce the three following subsets of functions belonging to $\mathcal{L}_{p}^{2}$ :

- Subset $\mathcal{C}_{2}$ [see (10)-(12) shown at the bottom of the page];

- Subset $\mathcal{C}_{3}$;

- Subset $\mathcal{C}_{4}$ with $L(\mathbf{y}, \eta, \theta)=(f(\mathbf{y}, \eta) / f(\mathbf{y}, \theta))$.

Theorem 1 shows that, although these four subsets are generated in a different manner, they are the same.

Theorem 1:

$$
\mathcal{C}_{1}=\mathcal{C}_{2}=\mathcal{C}_{3}=\mathcal{C}_{4}
$$

The Proof of Theorem 1 (13) is given in Appendix A.

Consequently, the MMSEE (6) (best Bayesian bound) is the solution of the following constrained optimization problem: $\forall \mathcal{C}_{i} i=1, \ldots, 4$

$$
\left\{\begin{array}{l}
\min _{v} \int_{\Theta} \int_{\Omega} v^{2}(\mathbf{y}, \theta) p(\mathbf{y}, \theta) d \mathbf{y} d \theta \\
\text { subject to } v(\mathbf{y}, \theta) \in \mathcal{C}_{i} .
\end{array}\right.
$$

\section{WEISS-WEINSTEIN FAMILY UNIFICATION}

In the light of the previous analysis, it appears a natural manner to introduce Bayesian bound lower than the MMSEE. Indeed, if $\mathcal{P}_{i}$ is a subset of $\mathcal{C}_{i}$, the solution of

$$
\left\{\begin{array}{l}
\min _{v} \int_{\Theta} \int_{\Omega} v^{2}(\mathbf{y}, \theta) p(\mathbf{y}, \theta) d \mathbf{y} d \theta \\
\text { subject to } v(\mathbf{y}, \theta) \in \mathcal{P}_{i}
\end{array}\right.
$$

will be also a lower bound of the MMSEE. In this paper, we will first show that an appropriate choice of $\mathcal{P}_{i}$ leads to the Bayesian bounds of the Weiss-Weinstein family. Second, we will show how this approach can be used in order to build new minimal

$$
\begin{aligned}
& \mathcal{C}_{2}=\left\{v(\mathbf{y}, \theta) \in \mathcal{L}_{p}^{2} / \forall f \in \mathcal{F}, \int_{\Theta} \int_{\Omega} v(\mathbf{y}, \theta) \frac{\partial f(\mathbf{y}, \theta)}{\partial \theta} d \mathbf{y} d \theta=\int_{\Theta} \int_{\Omega} f(\mathbf{y}, \theta) d \mathbf{y} d \theta\right\} \\
& \mathcal{C}_{3}=\left\{v(\mathbf{y}, \theta) \in \mathcal{L}_{p}^{2} / \begin{array}{c}
\forall f \in \mathcal{F}, \text { and } \iint_{\Theta} f(\mathbf{y}, \theta) d \mathbf{y} d \theta=1, \text { and } \forall h \text { such that } \theta+h \in \Theta \\
\iint_{\Theta} \int_{\Omega} v(\mathbf{y}, \theta)(f(\mathbf{y}, \theta+h)-f(\mathbf{y}, \theta)) d \mathbf{y} d \theta=h
\end{array}\right\} \\
& \mathcal{C}_{4}=\left\{v(\mathbf{y}, \theta) \in \mathcal{L}_{p}^{2} / \begin{array}{c}
\forall f \in \mathcal{F}, \text { and } \iint_{\Theta} f(\mathbf{y}, \theta) d \mathbf{y} d \theta=1, \text { and } \forall h \text { such that } \theta \pm h \in \Theta, \text { and } \forall s \in[0,1] \\
\iint_{\Omega} v(\mathbf{y}, \theta)\left[L^{s}(\mathbf{y}, \theta+h, \theta)-L^{1-s}(\mathbf{y}, \theta-h, \theta)\right] f(\mathbf{y}, \theta) d \mathbf{y} d \theta \\
=h \int_{\Theta} \int_{\Omega} L^{1-s}(\mathbf{y}, \theta-h, \theta) f(\mathbf{y}, \theta) d \theta d \mathbf{y}
\end{array}\right\} .
\end{aligned}
$$


bounds, particularly, by solving the following constrained optimization problem:

$$
\left\{\begin{array}{l}
\min _{v} \int_{\Theta} \int_{\Omega} v^{2}(\mathbf{y}, \theta) p(\mathbf{y}, \theta) d \mathbf{y} d \theta \\
\text { subject to } v(\mathbf{y}, \theta) \in \mathcal{P}_{i} \cap \mathcal{P}_{j} \quad i \neq j .
\end{array}\right.
$$

In this section, we restrict $\mathcal{C}_{2}, \mathcal{C}_{3}$, and $\mathcal{C}_{4}$ in order to obtain a general framework to create minimal bounds. Then, by way of a constrained optimization problem for which we give an explicit solution we unify the bounds of the Weiss-Weinstein family.

\section{A. A General Class of Lower Bounds Based on $\mathcal{C}_{2}, \mathcal{C}_{3}$, and $\mathcal{C}_{4}$}

Thanks to Theorem 1, we have proposed four equivalent sets of functions $v(\mathbf{y}, \theta)$ leading to the MMSEE. Note that this equivalence holds for (17)-(19), shown at the bottom of the page.

Consequently, if we take a finite set of functions $f(\mathbf{y}, \theta)$, a finite set of values $h$, and a finite set of values $s$, we will find bounds lower than the best Bayesian bounds and consequently a general class of minimal bounds on the MSE.

In this way, $\mathcal{C}_{2}, \mathcal{C}_{3}$, and $\mathcal{C}_{4}$ are restricted, respectively, as follows in (20)-(22), shown at the bottom of the page, with $L(\mathbf{y}, \eta, \theta)=(f(\mathbf{y}, \eta) / f(\mathbf{y}, \theta))$.
$\mathcal{P}_{2}, \mathcal{P}_{3}$, and $\mathcal{P}_{4}$ define a set of finite constraints, and (15) becomes a classical linear constrained optimization problem

$$
\left\{\begin{array}{l}
\min _{v} \int_{\Theta} \int_{\Omega} v^{2}(\mathbf{y}, \theta) p(\mathbf{y}, \theta) d \mathbf{y} d \theta \\
\text { subject to } \int_{\Theta} \int_{\Omega} v(\mathbf{y}, \theta) \tilde{g}_{k}(\mathbf{y}, \theta) d \mathbf{y} d \theta=c_{k} \quad k=1 \ldots K
\end{array}\right.
$$

where $g_{k}(\mathbf{y}, \theta)$, and $c_{k}$ are the functions and the scalars involved in $\mathcal{P}_{2}, \mathcal{P}_{3}$, and $\mathcal{P}_{4}$.

For $\mathcal{P}_{2}$

$$
\begin{aligned}
\tilde{g}_{k}(\mathbf{y}, \theta) & =\frac{\partial f_{k}(\mathbf{y}, \theta)}{\partial \theta} \\
c_{k} & =\int_{\Theta} \int_{\Omega} f_{k}(\mathbf{y}, \theta) d \mathbf{y} d \theta \quad \text { and } \\
K & =r .
\end{aligned}
$$

For $\mathcal{P}_{3}$

$$
\begin{aligned}
\tilde{g}_{k}(\mathbf{y}, \theta) & =f\left(\mathbf{y}, \theta+h_{k}\right)-f(\mathbf{y}, \theta) \\
c_{k} & =h_{k} \text { and } K=r .
\end{aligned}
$$

For $\mathcal{P}_{4}$, see (26) at the bottom of the page.

$$
\begin{aligned}
& \forall f(\mathbf{y}, \theta) \in \mathcal{F} \text { in the subset } \mathcal{C}_{2} \\
& \left\{\begin{array}{l}
\forall f(\mathbf{y}, \theta) \in \mathcal{F} \text { such that } \int_{\Theta} \int_{\Omega} f(\mathbf{y}, \theta) d \mathbf{y} d \theta=1 \\
\forall h \text { such that } \theta+h \in \Theta .
\end{array} \text { in the subset } \mathcal{C}_{3}\right. \\
& \left\{\begin{array}{l}
\forall f(\mathbf{y}, \theta) \in \mathcal{F} \text { such that } \iint_{\Theta} f(\mathbf{y}, \theta) d \mathbf{y} d \theta=1 \\
\forall h \text { such that } \theta \pm h \in \Theta, \\
\forall s \in[0,1] .
\end{array}\right.
\end{aligned}
$$

for a finite set of functions $f_{i}(\mathbf{y}, \theta) \in \mathcal{F}, \quad i=1 \cdots r$

$\mathcal{P}_{2}:\left\{\int_{\Theta} \int_{\Omega} v(\mathbf{y}, \theta) \frac{\partial f_{i}(\mathbf{y}, \theta)}{\partial \theta} d \mathbf{y} d \theta=\iint_{\Theta} f_{i}(\mathbf{y}, \theta) d \mathbf{y} d \theta\right.$.

$\mathcal{P}_{3}:\left\{\begin{array}{l}\text { for a particular function } f(\mathbf{y}, \theta) \in \mathcal{F} \text { such that } \int_{\Theta} \int_{\Omega} f(\mathbf{y}, \theta) d \mathbf{y} d \theta=1 \\ \text { for a finite set of value } h_{i} \text { such that } \theta+h_{i} \in \Theta, \quad i=1 \cdots r \\ \int_{\Theta} \int_{\Omega} v(\mathbf{y}, \theta)\left(f\left(\mathbf{y}, \theta+h_{i}\right)-f(\mathbf{y}, \theta)\right) d \mathbf{y} d \theta=h_{i} .\end{array}\right.$

$\mathcal{P}_{4}:\left\{\begin{array}{l}\text { for a particular function } f(\mathbf{y}, \theta) \in \mathcal{F} \text { such that } \int_{\Theta} \int_{\Omega} f(\mathbf{y}, \theta) d \mathbf{y} d \theta=1 \\ \text { for a finite set of value } h_{i} \text { such that } \theta+h_{i} \in \Theta, \quad i=1 \cdots r \\ \text { for a finite set of value } s_{i} \text { such that } s_{i} \in[0,1], \quad i=1 \cdots r \\ \int_{\Theta} \int_{\Omega} v(\mathbf{y}, \theta)\left[L^{s_{j}}\left(\mathbf{y}, \theta+h_{i}, \theta\right)-L^{1-s_{j}}\left(\mathbf{y}, \theta-h_{i}, \theta\right)\right] f(\mathbf{y}, \theta) d \mathbf{y} d \theta=h_{i} \int_{\Theta} \int_{\Omega} L^{1-s_{j}}\left(\mathbf{y}, \theta-h_{i}, \theta\right) f(\mathbf{y}, \theta) d \theta d \mathbf{y}\end{array}\right.$

$$
\left\{\begin{array}{l}
\tilde{g}_{k}(\mathbf{y}, \theta)=\left[L^{s_{k}}\left(\mathbf{y}, \theta+h_{k}, \theta\right)-L^{1-s_{k}}\left(\mathbf{y}, \theta-h_{k}, \theta\right)\right] f(\mathbf{y}, \theta) \\
c_{k}=h_{k} \int_{\Theta} \int_{\Omega}^{1-s_{k}}\left(\mathbf{y}, \theta-h_{k}, \theta\right) f(\mathbf{y}, \theta) d \theta d \mathbf{y} \\
\text { and } K=r .
\end{array}\right.
$$


Theorem 2 below gives the solution of (23). Note that this theorem has already been used in the case of a deterministic parameter in [17].

Theorem 2: Let $\mathbf{x} \in \mathbb{R}^{N}$ be a real vector and $p(\mathbf{x})$ and $q(\mathbf{x})$ be two functions of $\mathbb{R}^{N} \rightarrow \mathbb{R}$. Let

$$
\langle p(\mathbf{x}), q(\mathbf{x})\rangle=\int_{\mathbb{R}^{N}} p(\mathbf{x}) q(\mathbf{x}) d \mathbf{x}
$$

be an inner product of these two functions and its associate norm $\|p(\mathbf{x})\|^{2}=\langle p(\mathbf{x}), p(\mathbf{x})\rangle$. Let $u(\mathbf{x})$ and $g_{0}(\mathbf{x}), \ldots, g_{K}(\mathbf{x})$ be a set of functions of $\mathbb{R}^{N} \rightarrow \mathbb{R}$, and let $c_{0}, c_{1}, \ldots, c_{K}$ and $K+1$ be real numbers. Then, the solution of the constrained optimization problem leading to the minimum of $\|u(\mathbf{x})\|^{2}$ under the following $K+1$ constraints

$$
\left\langle u(\mathbf{x}), g_{k}(\mathbf{x})\right\rangle=c_{k} \quad k=0, \ldots, K
$$

is given by

$$
\left\{\begin{array}{l}
\min _{u}\|u(\mathbf{x})\|^{2}=\mathbf{c}^{T} \mathbf{G}^{-1} \mathbf{c} \\
\text { subject to }(28)
\end{array}\right.
$$

with

$$
\mathbf{c}=\left[\begin{array}{llll}
c_{0} & c_{1} & \cdots & c_{K}
\end{array}\right]^{T}
$$

and

$$
\{\mathbf{G}\}_{m, n}=\left\langle g_{m}(\mathbf{x}), g_{n}(\mathbf{x})\right\rangle .
$$

The Proof of Theorem 2 (29) is given in Appendix B.

\section{B. Application to the Weiss-Weinstein Family}

Using (29), $\mathcal{P}_{2}, \mathcal{P}_{3}$, and $\mathcal{P}_{4}$, we have built a general framework to obtain Bayesian minimal bounds on the MSE. In this section, we apply this framework and we revisit the Bayesian bounds of the Weiss-Weinstein family. Let $\mathbf{x}=\left[\begin{array}{ll}\mathbf{y}^{T} & \theta\end{array}\right]$ and $u(\mathbf{x})=v(\mathbf{y}, \theta) \sqrt{p(\mathbf{y}, \theta)}$ (i.e., $\tilde{g}_{k}(\mathbf{y}, \theta)=\sqrt{p(\mathbf{y}, \theta)} g_{k}(\mathbf{y}, \theta)$. Note that Theorem 2 still holds for a set of complex observations $\overline{\mathbf{y}}$ by letting $\mathbf{y}=\left[\operatorname{Re}\left\{\overline{\mathbf{y}}^{T}\right\} \quad \operatorname{Im}\left\{\overline{\mathbf{y}}^{T}\right\}\right]^{T}$.

Moreover, due to the restriction at some particular values of $f(\mathbf{y}, \theta), h$, and $s$, it is still possible to add constraints with our prior on the MMSEE in order to achieve tighter bounds. Here we will use the natural constraints of a null bias in terms of the joint probability function; i.e., $\int_{\Theta} \int_{\Omega} v(\mathbf{y}, \theta) p(\mathbf{y}, \theta) d \mathbf{y} d \theta=0$, where $p(\mathbf{y}, \theta)$ is the joint density of the problem (i.e., $g_{0}(\mathbf{y}, \theta)=$ $\sqrt{p(\mathbf{y}, \theta)}$ and $\left.c_{0}=0\right)$.

a) Bayesian Cramér-Rao Bound: By using the set $\mathcal{P}_{2}$ with $K=1$ and $f_{1}(\mathbf{y}, \theta)=p(\mathbf{y}, \theta)$ (consequently, $\left.\iint_{\Theta} f_{1}(\mathbf{y}, \theta) d \mathbf{y} d \theta=1\right)$, we obtain the following set of constraints:

$$
\left\{\begin{array}{l}
\mathbf{c}=\left[\begin{array}{ll}
0 & 1
\end{array}\right]^{T} \\
g_{0}(\mathbf{y}, \theta)=\sqrt{p(\mathbf{y}, \theta)} \\
g_{1}(\mathbf{y}, \theta)=\frac{1}{\sqrt{p(\mathbf{y}, \theta)}} \frac{\partial p(\mathbf{y}, \theta)}{\partial \theta} .
\end{array}\right.
$$

Matrix $\mathbf{G}$ involved in Theorem 2 is

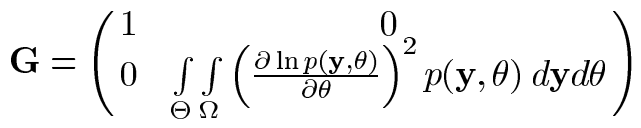

since $\int_{\Theta} \int_{\Omega}(\partial p(\mathbf{y}, \theta) / \partial \theta) d \mathbf{y} d \theta=0$.

Finally

$$
\begin{aligned}
\mathbf{c}^{T} \mathbf{G}^{-1} \mathbf{c} & =\left(\int_{\Theta} \int_{\Omega}\left(\frac{\partial \ln p(\mathbf{y}, \theta)}{\partial \theta}\right)^{2} p(\mathbf{y}, \theta) d \mathbf{y} d \theta\right)^{-1} \\
& =\text { BCRB }
\end{aligned}
$$

which is the Bayesian Cramér-Rao bound [20, pp. 72 and 84].

b) Bayesian Bhattacharyya Bound: By using the set $\mathcal{P}_{2}$ with $K=r$ and $f_{k}(\mathbf{y}, \theta)=\left(\partial^{k-1} p(\mathbf{y}, \theta) / \partial \theta^{k-1}\right)$, we obtain the following set of constraints:

$$
\left\{\begin{array}{l}
\mathbf{c}=\left[\begin{array}{lllll}
0 & 1 & 0 & \ldots & 0
\end{array}\right]^{T} \\
g_{0}(\mathbf{y}, \theta)=\sqrt{p(\mathbf{y}, \theta)} \\
g_{k}(\mathbf{y}, \theta)=\frac{1}{\sqrt{p(\mathbf{y}, \theta)}} \frac{\partial^{k} p(\mathbf{y}, \theta)}{\partial \theta^{k}} \quad k=1, \ldots, K .
\end{array}\right.
$$

We assume that the joint probability density function is such that $\lim _{\theta \rightarrow \pm \infty}\left(\partial^{k-1} p(\mathbf{y}, \theta) / \partial \theta^{k-1}\right)=0 \quad$ for $k=3, \ldots, K$. With this assumption and (9), we have

$$
\begin{aligned}
\mathbf{c}^{T} \mathbf{G}^{-1} \mathbf{c} & =\left\{\mathbf{B}^{-1}\right\}_{1,1} \\
& =\text { Bhatt } B
\end{aligned}
$$

where

$$
\{\mathbf{B}\}_{i, j}=\int_{\Theta} \int_{\Omega} \frac{1}{p(\mathbf{y}, \theta)} \frac{\partial^{i} p(\mathbf{y}, \theta)}{\partial \theta^{i}} \frac{\partial^{j} p(\mathbf{y}, \theta)}{\partial \theta^{j}} d \mathbf{y} d \theta
$$

which is the Bayesian Bhattacharyya bound [20, p. 149].

c) Bobrovsky-MayerWolf-Zakaï Bound: By using the set $\mathcal{P}_{2}$ with $K=1$ and $f_{1}(\mathbf{y}, \theta)=q(\mathbf{y}, \theta) p(\mathbf{y}, \theta)$, where $q(\mathbf{y}, \theta)$ is any function such that $f_{1}(\mathbf{y}, \theta)$ satisfies (9), we obtain the following set of constraints:

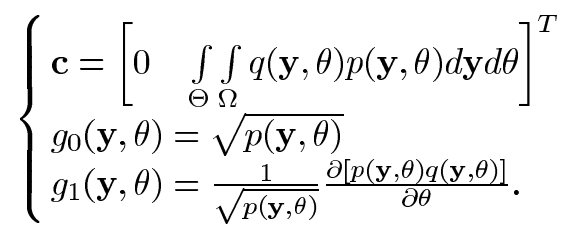

Due to (9), $\int_{\Theta} \int_{\Omega}(\partial q(\mathbf{y}, \theta) p(\mathbf{y}, \theta) / \partial \theta) d \mathbf{y} d \theta=0$ and the matrix $\mathbf{G}$ involved in Theorem 2 is

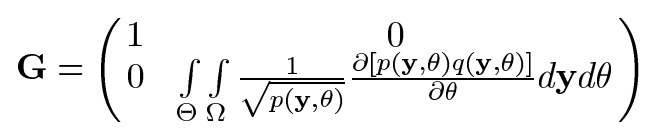

Finally

$$
\begin{aligned}
\mathbf{c}^{T} \mathbf{G}^{-1} \mathbf{c} & =\frac{\left(\iint_{\Theta} q(\mathbf{y}, \theta) p(\mathbf{y}, \theta) d \theta d \mathbf{y}\right)^{2}}{\int_{\Theta} \int_{\Omega} \frac{1}{p(\mathbf{y}, \theta)}\left(\frac{\partial[p(\mathbf{y}, \theta) q(\mathbf{y}, \theta)]}{\partial \theta}\right)^{2} d \mathbf{y} d \theta} \\
& =\stackrel{\operatorname{BMZB}(q(\mathbf{y}, \theta)) .}{ }
\end{aligned}
$$

We recognize the Bobrovsky-MayerWolf-Zakaï bound [39], which is an extension of the Bayesian Cramér-Rao bound, since

$$
\operatorname{BMZB}(1)=\text { BCRB. }
$$


d) Bobrovsky-Zakaï Bound: We choose here that the particular value of $f(\mathbf{y}, \theta)=p(\mathbf{y}, \theta)$, the joint density probability function of the problem. Consequently, $\iint_{\Omega} f(\mathbf{y}, \theta) d \mathbf{y} d \theta=1$.

By using the set $\mathcal{P}_{3}$ with $K=1$, we obtain the following set of constraints:

$$
\left\{\begin{array}{l}
\mathbf{c}=\left[\begin{array}{ll}
0 & h
\end{array}\right]^{T} \\
g_{0}(\mathbf{y}, \theta)=\sqrt{p(\mathbf{y}, \theta)} \\
g_{1}(\mathbf{y}, \theta)=\frac{p(\mathbf{y}, \theta+h)-p(\mathbf{y}, \theta)}{\sqrt{p(\mathbf{y}, \theta)}}
\end{array}\right.
$$

Matrix $\mathbf{G}$ involved in Theorem 2 is

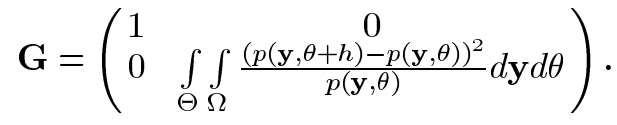

Finally

$$
\begin{aligned}
\mathbf{c}^{T} \mathbf{G}^{-1} \mathbf{c} & =\frac{h^{2}}{\int_{\Theta} \int_{\Omega} \frac{p^{2}(\mathbf{y}, \theta+h)}{p(\mathbf{y}, \theta)} d \mathbf{y} d \theta-1} \\
& =\operatorname{BZB}(h) .
\end{aligned}
$$

Since $h$ is a parameter left to the user, the highest bound that can be obtained with (44) is given by

$$
\mathrm{BZB}=\sup _{h} \mathrm{BZB}(h)=\sup _{h} \frac{h^{2}}{\iint_{\Theta} \frac{p^{2}(\mathbf{y}, \theta+h)}{p(\mathbf{y}, \theta)} d \mathbf{y} d \theta-1}
$$

which is the Bobrovsky-Zakaï bound [40].

e) Reuven-Messer Bound: We choose here that the particular value of $f(\mathbf{y}, \theta)=p(\mathbf{y}, \theta)$, the joint density probability function of the problem. Consequently, $\iint_{\Omega} f(\mathbf{y}, \theta) d \mathbf{y} d \theta=1$.

In order to obtain a bound tighter than the Bobrovsky-Zakai bound (i.e., $\mathcal{P}_{3} \rightarrow \mathcal{C}_{3}$ ), we use the set $\mathcal{P}_{3}$ with $K=r$. We then obtain the following set of constraints:

$$
\left\{\begin{array}{l}
\mathbf{c}=\left[\begin{array}{ll}
0 & \mathbf{h}^{T}
\end{array}\right]^{T} \\
g_{0}(\mathbf{y}, \theta)=\sqrt{p(\mathbf{y}, \theta)} \\
g_{k}(\mathbf{y}, \theta)=\frac{p\left(\mathbf{y}, \theta+h_{k}\right)-p(\mathbf{y}, \theta)}{\sqrt{p(\mathbf{y}, \theta)}} \quad k=1, \ldots, r
\end{array}\right.
$$

where $\mathbf{h}=\left[\begin{array}{lll}h_{1} & \cdots & h_{r}\end{array}\right]^{T}$.

Matrix $\mathbf{G}$ involved in Theorem 2 is

$$
\mathbf{G}=\left(\begin{array}{cccc}
1 & 0 & \cdots & 0 \\
0 & & & \\
\vdots & & \mathbf{D} & \\
0 & & &
\end{array}\right)
$$

where $\mathbf{D}(r \times r)$ is defined as shown in (48) shown at the bottom of the page.

Finally

$$
\begin{aligned}
\mathbf{c}^{T} \mathbf{G}^{-1} \mathbf{c} & =\mathbf{h}^{T} \mathbf{D}^{-1} \mathbf{h} \\
& =\operatorname{RMB}(\mathbf{h}) .
\end{aligned}
$$

As for the Bobrovsky-Zakaï bound, since $\mathbf{h}$ is a parameter vector left to the user, the highest bound that can be obtained with (49) is given by

$$
\mathrm{RMB}=\sup _{\mathbf{h}} \operatorname{RMB}(\mathbf{h})=\sup _{\mathbf{h}} \mathbf{h}^{T} \mathbf{D}^{-1} \mathbf{h}
$$

which is a particular case ${ }^{1}$ of the Reuven-Messer bound [41].

f) Weiss-Weinstein Bound: We choose here that the particular value of $f(\mathbf{y}, \theta)=p(\mathbf{y}, \theta)$, the joint density probability function of the problem. Consequently, $\iint_{\Omega} f(\mathbf{y}, \theta) d \mathbf{y} d \theta=1$.

By using the set $\mathcal{P}_{4}$ with $K=r$, we obtain the following set of constraints: [see (51) at the bottom of the next page].

Let, [see (52)-(54) at the bottom of the next page].

The application of Theorem 2 leads to

$$
\begin{aligned}
\mathbf{c}^{T} \mathbf{G}^{-1} \mathbf{c} & =\boldsymbol{\xi}^{T} \mathbf{W}^{-1} \boldsymbol{\xi} \\
& =\operatorname{WWB}(\mathbf{h}, \mathbf{s})
\end{aligned}
$$

where

$$
\begin{aligned}
\{\mathbf{W}\}_{i, j}= & \mathbb{E}_{\mathbf{y}, \theta}\left[\left(L^{s_{i}}\left(\mathbf{y}, \theta+h_{i}, \theta\right)\right.\right. \\
& \left.-L^{1-s_{i}}\left(\mathbf{y}, \theta-h_{i}, \theta\right)\right)\left(L^{s_{j}}\left(\mathbf{y}, \theta+h_{j}, \theta\right)\right. \\
& \left.\left.-L^{1-s_{j}}\left(\mathbf{y}, \theta-h_{j}, \theta\right)\right)\right] .
\end{aligned}
$$

As for the Bobrovsky-Zakaï bound and the Reuven-Messer bound, since $\mathbf{h}$ and $\mathbf{s}$ are parameter vectors left to the user, the highest bound that can be obtained with (55) is given by

$$
\mathrm{WWB}=\sup _{\mathbf{h}, \mathbf{s}} \mathrm{WWB}(\mathbf{h}, \mathbf{s})=\sup _{\mathbf{h}, \mathbf{s}} \boldsymbol{\xi}^{T} \mathbf{W}^{-1} \boldsymbol{\xi}
$$

We recognize the Weiss-Weinstein bound [42].

\section{New Minimal BoundS}

The framework proposed in the last section allows us to rederive all the bounds of the Weiss-Weinstein family by way of a constrained optimization problem. But this framework is also

${ }^{1}$ In 1997, Reuven and Messer proposed a hybrid minimal bound based on the Barankin bound for both random and nonrandom vector of parameters. Here, only the random case is considered.

$$
\begin{aligned}
\{\mathbf{D}\}_{i, j} & =\int_{\Theta} \int_{\Omega} \frac{\left(p\left(\mathbf{y}, \theta+h_{i}\right)-p(\mathbf{y}, \theta)\right)\left(p\left(\mathbf{y}, \theta+h_{j}\right)-p(\mathbf{y}, \theta)\right)}{p(\mathbf{y}, \theta)} d \mathbf{y} d \theta \\
& =\int_{\Theta} \int_{\Omega} \frac{p\left(\mathbf{y}, \theta+h_{i}\right) p\left(\mathbf{y}, \theta+h_{j}\right)}{p(\mathbf{y}, \theta)} d \mathbf{y} d \theta-1 .
\end{aligned}
$$


useful for deriving new lower bounds. In this section, we propose two lower bounds.

\section{A. Some Global Classes of Bhattacharyya Bounds}

In [39], Bobrovsky, Mayer-Wolf, and Zakaï propose an extension of the Bayesian Cramér-Rao bound given by (40). The advantage of this bound is the degree of freedom given by $q(\mathbf{y}, \theta)$. Indeed, the authors give some examples for which use of a properly chosen function $q(\mathbf{y}, \theta)$ leads to useful bounds. Moreover, when $p(\mathbf{y}, \theta)$ does not satisfy the regularity assumption given in [20] (e.g., for uniform random variables), a properly chosen $q(\mathbf{y}, \theta)$ can solve the problem. Here we obtain an extension of this bound and of the Bayesian Bhattacharyya bound in a straightforward manner by mixing the constraints of the Bobrovsky-MayerWolf-Zakaï bound and the constraints of the Bayesian Bhattacharyya bound.

By using the set $\mathcal{P}_{2}$ with $K=r$ and $f_{k}(\mathbf{y}, \theta)=$ $\left(\partial^{k-1}[q(\mathbf{y}, \theta) p(\mathbf{y}, \theta)] / \partial \theta^{k-1}\right)$, where $q(\mathbf{y}, \theta)$ is any function such that $f_{k}(\mathbf{y}, \theta)$ satisfies $(9)$, we obtain the following set of constraints:

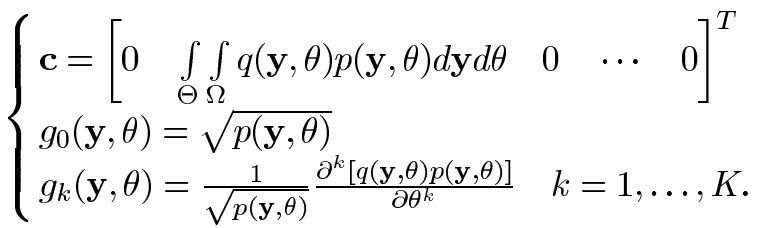

We assume that the functions $q(\mathbf{y}, \theta)$ and $p(\mathbf{y}, \theta)$ are such that $\lim _{\theta \rightarrow \pm \infty}\left(\partial^{k-1} q(\mathbf{y}, \theta) p(\mathbf{y}, \theta) / \partial \theta^{k-1}\right)=0$ for $k=3, \ldots, K$. With this assumption and (9), we have

$$
\mathbf{c}^{T} \mathbf{G}^{-1} \mathbf{c}=\frac{\left(\int_{\Theta} \int_{\Omega} q(\mathbf{y}, \theta) p(\mathbf{y}, \theta) d \mathbf{y} d \theta\right)^{2}}{\left\{\overline{\mathbf{B}}^{-1}\right\}_{1,1}}
$$

where [see (60) shown at the bottom of the page].

\section{B. The Bayesian Abel Bound}

In this section, we propose a new minimal bound on the MSE based on our framework and on the Abel works on deterministic bounds [29], [30]. In the deterministic parameter context, the Cramér-Rao bound and the Bhattacharyya bound account for the small estimation error (near the true value of the parameters). The Chapman-Robbins bound and the Barankin bound account for the large estimation error generally due to the appearance of outliers which creates the performance breakdown phenomena. In [29] and [30], Abel combined the two kinds of bounds in order to obtain a bound that accounts for both local and large errors. The obtained deterministic Abel bound leads to a generalization of the Cramér-Rao, the Bhattacharyya, the Chapman-Robbins, and the Barankin bounds. As the deterministic bounds, the Bayesian Cramér-Rao bound and the Bayesian Bhattacharyya bound are small error bounds, as compared to the Bobrovsky-Zakaï bound and the Reuven-Messer bound which are large error bounds. The purpose here is to apply the idea of Abel in the Bayesian context, i.e., to derive a bound that combines the Bayesian small and large error bounds. This application will be accomplished by way of the constrained optimization problem introduced in the last section. Our Bayesian version of the Abel bound is derived by mixing the constraints of the Reuven-Messer bound and the Bayesian Bhattacharyya bound and, thus, represents a generalization of these bounds. Consequently, we are solving the following constrained optimization problem

$$
\left\{\begin{array}{l}
\min _{v} \int_{\Theta} \int_{\Omega} v^{2}(\mathbf{y}, \theta) p(\mathbf{y}, \theta) d \mathbf{y} d \theta \\
\text { subject to } v(\mathbf{y}, \theta) \in \mathcal{P}_{2} \cap \mathcal{P}_{3} .
\end{array}\right.
$$

By combining the Bayesian Bhattacharyya constraints (35) and the Reuven-Messer constraints (46), i.e., by concatenating both vectors $\mathbf{g}=\left[g_{0}(\mathbf{y}, \theta), g_{1}(\mathbf{y}, \theta), \ldots, g_{K}(\mathbf{y}, \theta)\right]^{T}$ and $\mathbf{c}$ from the Bayesian Bhattacharyya bound of order $m$ and from

$$
\left\{\begin{array}{l}
\mathbf{c}=\left[0 \quad h_{1} \mathbb{E}_{\mathbf{y}, \theta}\left[L^{1-s_{1}}\left(\mathbf{y}, \theta-h_{1}, \theta\right)\right] \quad \cdots \quad h_{k} \mathbb{E}_{\mathbf{y}, \theta}\left[L^{1-s_{k}}\left(\mathbf{y}, \theta-h_{k}, \theta\right)\right]\right]^{T} \\
g_{0}(\mathbf{y}, \theta)=\sqrt{p(\mathbf{y}, \theta)} \\
g_{k}(\mathbf{y}, \theta)=\sqrt{p(\mathbf{y}, \theta)}\left(L^{s_{k}}\left(\mathbf{y}, \theta+h_{k}, \theta\right)-L^{1-s_{k}}\left(\mathbf{y}, \theta-h_{k}, \theta\right)\right) \quad k=1, \ldots, r .
\end{array}\right.
$$

$$
\begin{aligned}
\xi & =\left[\begin{array}{lll}
h_{1} \mathbb{E}_{\mathbf{y}, \theta}\left[L^{1-s_{1}}\left(\mathbf{y}, \theta-h_{1}, \theta\right)\right] & \cdots & h_{r} \mathbb{E}_{\mathbf{y}, \theta}\left[L^{1-s_{r}}\left(\mathbf{y}, \theta-h_{r}, \theta\right)\right.
\end{array}\right]^{T} \\
\mathbf{h} & =\left[\begin{array}{lll}
h_{1} & \cdots & h_{r}
\end{array}\right]^{T} \\
\mathbf{s} & =\left[\begin{array}{lll}
s_{1} & \cdots & s_{r}
\end{array}\right]^{T} .
\end{aligned}
$$


the Reuven-Messer bound of order $r$, we obtain the following new set of $K=m+r+1$ constraints ${ }^{2}$ :

$$
\mathbf{g}=\frac{1}{\sqrt{p(\mathbf{y}, \theta)}}\left[\begin{array}{c}
p(\mathbf{y}, \theta) \\
\frac{\partial p(\mathbf{y}, \theta)}{\partial \theta} \\
\vdots \\
\frac{\partial^{m} p(\mathbf{y}, \theta)}{\partial \theta^{m}}-- \\
--\frac{\left.\mathbf{y}, \theta+h_{1}\right)-p(\mathbf{y}, \theta)}{\vdots} \\
p\left(\mathbf{y}, \theta+h_{r}\right)-p(\mathbf{y}, \theta)
\end{array}\right]
$$

and

$$
\mathbf{c}=\left[\begin{array}{c}
0 \\
1 \\
0 \\
\vdots \\
0 \\
-- \\
h_{1} \\
\vdots \\
h_{r}
\end{array}\right]
$$

The calculus are detailed in Appendix C, and the Theorem 2 leads to

$$
\mathbf{c}^{T} \mathbf{G}^{-1} \mathbf{c}=\mathrm{BAB}_{m, r}(\mathbf{h})=\boldsymbol{\alpha}^{T} \mathbf{B}^{-1} \boldsymbol{\alpha}+\mathbf{u}^{T} \mathbf{J}^{-1} \mathbf{u}
$$

with

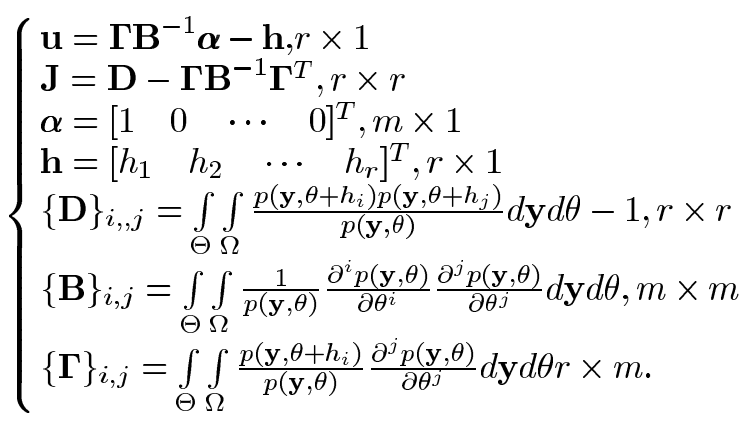

Let us note that the first term on right-hand side (RHS) of (63) is equal to $\mathrm{BAB}_{m, 0}$, which is the Bayesian Bhattacharyya bound of order $m$, and that $\mathrm{BAB}_{0, r}(\mathbf{h})$ is the Reuven-Messer bound of order $r$. We have previously shown that problem (8) leads to the MMSEE (the best Bayesian bound). Here, from the increase of constraints, it follows that the Bayesian Abel bound is (for $r$ and $m$ fixed) a better approximation of the best Bayesian bound than the Bayesian Bhattacharyya bound of order $m$ and the Reuven-Messer bound of order $r$.

The Bayesian Abel bound as the Reuven-Messer bound depends on $r$ free parameters $h_{1}, \ldots, h_{r}$. Then, a maximization over these parameters is desired to obtain the highest bound. Therefore, the best Bayesian Abel bound is given by

$$
\mathrm{BAB}_{m, r}=\sup _{\mathbf{h}}\left(\boldsymbol{\alpha}^{T} \mathbf{B}^{-1} \boldsymbol{\alpha}+\mathbf{u}^{T} \mathbf{J}^{-1} \mathbf{u}\right) .
$$

This multidimensional optimization brings with it a huge computational cost. A possible alternative is given by noting that the Bayesian Cramér-Rao bound is a particular case of the Bayesian Bhattacharyya bound (single derivative) and that the Bobrovsky-Zakaï bound is a particular case of the Reuven-Messer bound (single test point). Therefore, finding a tractable form of the Bayesian Abel bound in the case where $m=1$ and $r=1$ could be interesting, since the obtained bound will be tighter than both the Bayesian Cramér-Rao bound and the Bobrovsky-Zakaï bound with a low computational cost. In this case, (65) becomes straightforwardly

$$
\mathrm{BAB}_{1,1}=\sup _{h} \frac{\mathrm{BCRB}^{-1}+\mathrm{BZB}^{-1}(h)-2 \phi(h)}{\mathrm{BCRB}^{-1} \mathrm{BZB}^{-1}(h)-\phi^{2}(h)}
$$

where BCRB is the Bayesian Cramér-Rao bound, BZB is the Bobrovsky-Zakaï bound, and

$$
\phi(h)=\frac{1}{h} \int_{\Theta} \int_{\Omega} \frac{\partial \ln p(\mathbf{y}, \theta)}{\partial \theta} p(\mathbf{y}, \theta+h) d \mathbf{y} d \theta .
$$

Equation (66) is interesting, since if the Bayesian Cramér-Rao bound and the Bobrovsky-Zakaï bound are available for a given problem, the evaluation of the $\mathrm{BAB}_{1,1}$ requires only the computation of $\phi(h)$.

\section{Bayesian Bounds for Signal Processing Problems}

In this section, we illustrate our previous analysis through a spectral analysis problem. First, we propose several closedform expressions for the different bounds of the Weiss-Weinstein family (including the proposed Bayesian Abel bound) for a general Gaussian observation model with parameterized mean widely used in the signal processing literature (see, e.g., [68, p. 35]). Then, we apply these results to the spectral analysis problem. Finally, we give simulation results that compare the different bounds and show the superiority of the Weiss-Weinstein bound.

\section{A. Gaussian Observation Model With Parameterized Mean}

We consider the following general observation model:

$$
\mathbf{y}=\mathbf{m}(\theta)+\mathbf{n}
$$

where $\mathbf{y}$ is the complex observation vector $(N \times 1), \theta$ is a real unknown parameter, $\mathbf{m}$ is a complex deterministic vector $(N \times 1)$ depending (nonlinearly) on $\theta$, and $\mathbf{n}$ is the complex vector $(N \times 1)$ of the noise. The noise is assumed to be circular, Gaussian, with zero mean and with covariance matrix $\sigma^{2} \mathbf{I}_{N}$. The parameter of interest $\theta$ is assumed to have a Gaussian a priori probability density function with mean $\mu$ and variance $\sigma_{\theta}^{2}$

$$
p(\theta)=\frac{1}{\sqrt{2 \pi} \sigma_{\theta}} e^{-\frac{1}{2 \sigma_{\theta}^{2}}(\theta-\mu)^{2}} .
$$

For this model, the likelihood of the observations is given by

$$
p(\mathbf{y} \mid \theta)=\frac{1}{\left(\pi \sigma^{2}\right)^{N}} e^{-\frac{1}{\sigma^{2}}(\mathbf{y}-\mathbf{m}(\theta))^{H}(\mathbf{y}-\mathbf{m}(\theta))} .
$$


To the best of our knowledge, only the Cramér-Rao bound expression is known in this case (see [68]).

The Bayesian Bhattacharyya bound requires the calculation of several derivatives of the joint probability function in order to be significantly tighter than the Cramér-Rao bound, which is generally difficult (see $[69$, Ch. 4] for an example for which the Bhattacharyya bound of order 2 requires much algebraic effort to finally be equal to the Cramér-Rao bound). Consequently, we will not use this bound here.

The details are given in Appendix D.

1) Bayesian Cramér-Rao Bound:

$$
\mathrm{BCRB}=\frac{\sigma_{\theta}^{2}}{\frac{2 \sigma_{\theta}^{2}}{\sigma^{2}} \mathbb{E}_{\theta}\left[\left\|\frac{\partial \mathbf{m}(\theta)}{\partial \theta}\right\|^{2}\right]+1} .
$$

2) Bobrovsky-Zakaï Bound:

$$
\mathrm{BZB}=\sup _{h} \frac{h^{2}}{\int_{\Theta} \frac{p^{2}(\theta+h)}{p(\theta)} e^{\frac{2}{\sigma^{2}}\|\mathbf{m}(\theta+h)-\mathbf{m}(\theta)\|^{2}} d \theta-1} .
$$

3) Bayesian Abel Bound: $\mathrm{BAB}_{1,1}$ is given by (66):

$$
\mathrm{BAB}_{1,1}=\sup _{h} \frac{\mathrm{BCRB}^{-1}+\mathrm{BZB}^{-1}(h)-2 \phi(h)}{\mathrm{BCRB}^{-1} \mathrm{BZB}^{-1}(h)-\phi^{2}(h)}
$$

where

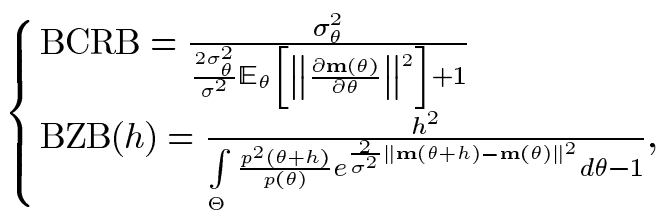

and

$$
\phi(h)=\frac{1}{\sigma_{\theta}^{2}}+\frac{2}{h \sigma^{2}} \mathbb{E}_{\theta+h}\left[\operatorname{Re}\left\{\frac{\partial \mathbf{m}^{H}(\theta)}{\partial \theta}(\mathbf{m}(\theta+h)-\mathbf{m}(\theta))\right\}\right] .
$$

4) Weiss-Weinstein Bound: We now consider the WeissWeinstein bound with one test point, which can be simplified as follows (see [42, eq. (6)]):

$$
\mathrm{WWB}=\sup _{h, s} \frac{h^{2} e^{2 \eta(s, h)}}{e^{\eta(2 s, h)}+e^{\eta(2-2 s,-h)}-2 e^{\eta(s, 2 h)}}
$$

where the key point to evaluate this bound is $\eta(\alpha, \beta)$, which is the semi-invariant moment generating function [70], defined as follows:

$$
\eta(\alpha, \beta)=\ln \int_{\Theta} \int_{\Omega} \frac{p^{\alpha}(\mathbf{y}, \theta+\beta)}{p^{\alpha-1}(\mathbf{y}, \theta)} d \mathbf{y} d \theta .
$$

This function is given by (78), as shown at the bottom of the page.

\section{B. Spectral Analysis Problem}

We now consider the following observation model involved in spectral analysis:

$$
y_{k}=a e^{j 2 \pi k \theta}+n_{k}, \quad k=0, \ldots, N-1
$$

where $y_{k}$ is the $k^{\text {th }}$ complex observation. The observations are assumed to be independent. $a$ is the amplitude of the single cisoïde of frequency $\theta .\left\{n_{k}\right\}$ is a sequence of random variables assumed complex, circular, i.i.d, Gaussian, with zero mean and variance $\sigma^{2}$. Consequently the SNR is given by $\mathrm{SNR}=\left(a^{2} / \sigma^{2}\right)$. The parameter of interest is the frequency $\theta \in \Theta=(-(1 / 2),(1 / 2)]$ which is a Gaussian random variable with mean $\mu$ and variance $\sigma_{\theta}^{2}$ (69).

This model is a particular case of the model (68), where

$$
\mathbf{m}(\theta)=a \mathbf{s}(\theta)
$$

with

$$
\mathbf{s}(\theta)=\left[\begin{array}{llll}
1 & e^{j 2 \pi \theta} & \cdots & e^{j 2 \pi(N-1) \theta}
\end{array}\right]^{T} .
$$

Let $\mathbf{y}=\left[\begin{array}{lll}y_{0} & \cdots & y_{N-1}\end{array}\right]^{T}$. The likelihood of the observation is given by

$$
\begin{aligned}
p(\mathbf{y} \mid \theta) & =\prod_{k=0}^{N-1} p\left(y_{k} \mid \theta\right) \\
& =\frac{1}{\left(\pi \sigma^{2}\right)^{N}} e^{-\frac{1}{\sigma^{2}}\left(\|\mathbf{y}\|^{2}-2 a \operatorname{Re}\left\{\sum_{k=0}^{N-1} y_{k}^{*} e^{j 2 \pi k \theta}\right\}+N a^{2}\right) .}
\end{aligned}
$$

Note that, if $\theta$ is assumed to be deterministic and in a digital communications context, some closed-form expressions of deterministic bounds can be found in [56].

The details of the calculus for the Weiss-Weinstein family are given in Appendix E.

1) Cramér-Rao Bound:

$$
\mathrm{BCRB}=\frac{\sigma_{\theta}^{2}}{\operatorname{SNR} \frac{4 \pi^{2} \sigma_{\theta}^{2}}{3} N(2 N-1)(N-1)+1} .
$$

2) Bobrovsky-Zakaï Bound:

$$
\mathrm{BZB}=\sup _{h} \frac{h^{2}}{e^{4 \mathrm{SNR}\left(N-\sin ^{2}(\pi h N)-\frac{1}{2} \frac{\sin (2 \pi h N)}{\tan (\pi h)}\right)+\frac{h^{2}}{\sigma_{\theta}^{2}}-1}} .
$$

3) Bayesian Abel Bound: The $\mathrm{BAB}_{1,1}$ is given by (66)

$$
\mathrm{BAB}_{1,1}=\sup _{h} \frac{\mathrm{BCRB}^{-1}+\mathrm{BZB}^{-1}(h)-2 \phi(h)}{\mathrm{BCRB}^{-1} \mathrm{BZB}^{-1}(h)-\phi^{2}(h)}
$$

$$
\eta(\alpha, \beta)=\ln \frac{1}{\sqrt{2 \pi} \sigma_{\theta}} \int_{\Theta} e^{\frac{\alpha(\alpha-1)}{\sigma^{2}}\|\mathbf{m}(\theta+\beta)-\mathbf{m}(\theta)\|^{2}-\frac{1}{2 \sigma_{\theta}^{2}}(\theta-(\sqrt{\alpha(\alpha-1)}-\alpha) h-\mu)(\theta+(\sqrt{\alpha(\alpha-1)}+\alpha) h-\mu)} d \theta .
$$


where

$$
\left\{\begin{array}{l}
\mathrm{BCRB}=\frac{1}{\operatorname{SNR}} \frac{\sigma_{\theta}^{2}}{\frac{4 \pi^{2} \sigma_{\theta}^{2}}{3} N(2 N-1)(N-1)+1} \\
\mathrm{BZB}(h)=\frac{h^{2}}{e^{4 \operatorname{SNR}\left(N-\sin ^{2}(\pi h N)-\frac{1}{2} \frac{\sin (2 \pi h N)}{\tan (\pi h)}\right)+\frac{h^{2}}{\sigma_{\theta}^{2}}-1}}
\end{array}\right.
$$

and

$$
\begin{array}{r}
\phi(h)=\frac{1}{\sigma_{\theta}^{2}}+\frac{2 \pi \mathrm{SNR}}{h}\left(N \frac{\cos (2 \pi h N)}{\tan (\pi h)}\right. \\
\left.-\sin (2 \pi h N)\left(\frac{1}{2 \sin (\pi h)}+N\right)\right) .
\end{array}
$$

4) Weiss-Weinstein Bound: The Weiss-Weinstein bound is given by

$$
\mathrm{WWB}=\sup _{h, s} \frac{h^{2} e^{2 \eta(s, h)}}{e^{\eta(2 s, h)}+e^{\eta(2-2 s,-h)}-2 e^{\eta(s, 2 h)}}
$$

where $\eta(\alpha, \beta)$ is given by

$$
\begin{aligned}
\eta(\alpha, \beta)=\alpha & (\alpha-1)(2 \operatorname{SNR}(N \\
& \left.\left.-\sin ^{2}(\pi \beta N)-\frac{1}{2} \frac{\sin (2 \pi \beta N)}{\tan (\pi \beta)}\right)-\frac{\beta^{2}}{2 \sigma_{\theta}^{2}}\right) .
\end{aligned}
$$

The Weiss-Weinstein bound needs to be optimized over two continuous parameters, which creates significant computational cost. Here, two methods for reducing the computational cost are presented.

- As previously stated, $h$ is chosen on the parameter support which is approximated by $\left[-3 \sigma_{\theta}, 3 \sigma_{\theta}\right]$. This support can be reduced to $\left[0,3 \sigma_{\theta}\right]$, since the function is even with respect to $h$. Note that this remark holds for the Bayesian Abel bound and the Bobrovsky-Zakaï bound.

- As proposed by Weiss and Weinstein in [42], it is sometimes a good choice to set $s=1 / 2$. This approximation is intuitively justified by the fact that the Weiss-Weinstein bound tends to the Bobrovsky-Zakaï bound when $s$ tends to zero or one. Unfortunately, no sound proof that this result is true in general is available in the literature. If we set $s=1 / 2, \eta(\alpha, \beta)$ is modified as follows: [see (90) at the bottom of the page] and the modified Weiss-Weinstein bound becomes

$$
\begin{aligned}
& \overline{\text { WWB }} \\
& =\sup _{h} \frac{h^{2}}{2} \frac{e^{-\frac{1}{2}\left(2 \operatorname{SNR}\left(N-\sin ^{2}(\pi h N)-\frac{1}{2} \frac{\sin (2 \pi h N)}{\tan (\pi h)}\right)-\frac{h^{2}}{2 \sigma_{\theta}^{2}}\right)}}{1-e^{-\frac{1}{2}\left(\operatorname{SNR}\left(N-\sin ^{2}(2 \pi h N)-\frac{1}{2} \frac{\sin (4 \pi h N)}{\tan (2 \pi h)}\right)-\frac{h^{2}}{\sigma_{\theta}^{2}}\right)}} .
\end{aligned}
$$

The resulting bound has approximaely the same computational cost as the BZB and the BAB.

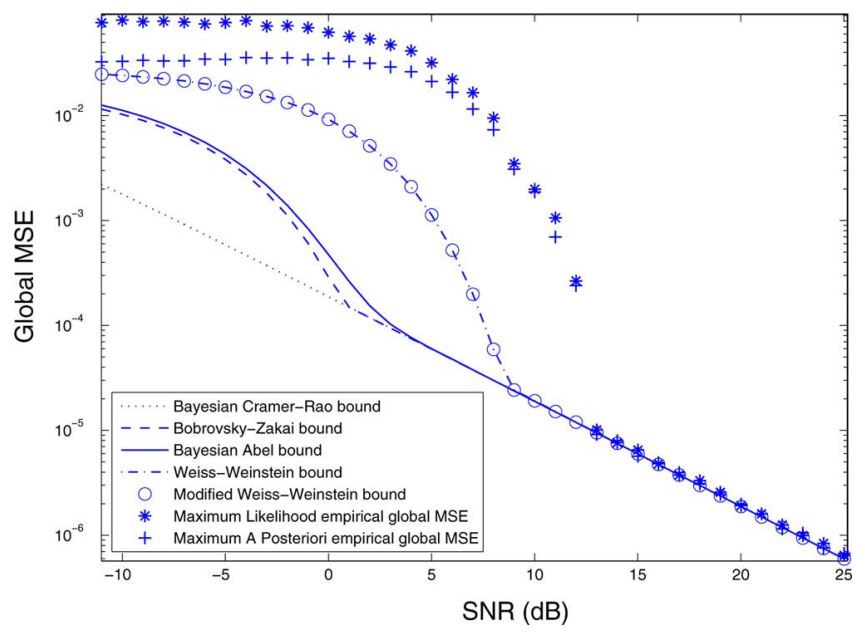

Fig. 1. Comparison of the global MSE of the MLE and of the MAP estimator, the Cramér-Rao bound, the Bobrovsky-Zakaï bound, the Bayesian Abel bound, and the Weiss-Weinstein bound with optimization over $s$ and $s=1 / 2 . N=15$ observations. $\sigma_{\theta}^{2}=1 / 36 \mathrm{rad}^{2}$.

\section{Simulations}

In order to illustrate our results on the different bounds, we present here a simulation result for the spectral analysis problem.

We consider a scenario with $N=15$ observations and, without loss of generality, $a=1$. The estimator will be the maximum likelihood estimator (MLE) given for this model by

$$
\hat{\theta}_{\mathrm{ML}}=\arg \min _{\theta}\left[\|\mathbf{y}\|^{2}+N a^{2}-2 a \operatorname{Re}\left\{\sum_{k=0}^{N-1} y_{k}^{*} e^{j 2 \pi k \theta}\right\}\right] \text {. }
$$

We also use the maximum a posteriori (MAP) estimator given by

$$
\begin{aligned}
\hat{\theta}_{\mathrm{MAP}}=\arg \min _{\theta} & {\left[\frac { 1 } { \sigma ^ { 2 } } \left(\|\mathbf{y}\|^{2}+N a^{2}\right.\right.} \\
& \left.\left.-2 a \operatorname{Re}\left\{\sum_{k=0}^{N-1} y_{k}^{*} e^{j 2 \pi k \theta}\right\}\right)+\frac{\theta^{2}}{2 \sigma_{\theta}^{2}}\right] .
\end{aligned}
$$

The global MSE will be computed by using (3) and 1000 Monte Carlo runs. For the a priori probability density function of the parameter of interest, we choose $\mu=0$ and $\sigma_{\theta}^{2}=(1 / 36)$ $\operatorname{rad}^{2}$.

Fig. 1 superimposes the global MSE of the MLE and of the MAP estimator, the Cramér-Rao bound, the Bobrovsky-Zakaï bound, the Bayesian Abel bound, and the Weiss-Weinstein bound with optimization over $s$ and $s=1 / 2$.

$$
\left\{\begin{array}{l}
\eta\left(\frac{1}{2}, h\right)=-\frac{1}{4}\left(2 \operatorname{SNR}\left(N-\sin ^{2}(\pi h N)-\frac{1}{2} \frac{\sin (2 \pi h N)}{\tan (\pi h)}\right)-\frac{h^{2}}{2 \sigma_{\theta}^{2}}\right) \\
\eta(1, h)=0 \\
\eta(1,-h)=0 \\
\eta\left(\frac{1}{2}, 2 h\right)=-\frac{1}{2}\left(\operatorname{SNR}\left(N-\sin ^{2}(2 \pi h N)-\frac{1}{2} \frac{\sin (4 \pi h N)}{\tan (2 \pi h)}\right)-\frac{h^{2}}{\sigma_{\theta}^{2}}\right)
\end{array}\right.
$$


This figure shows the threshold behavior of both estimators when the SNR decreases. In contrast to the Cramér-Rao bound, the Bobrovsky-Zakaï bound, the Bayesian Abel bound, and the Weiss-Weinstein bound exhibit the threshold phenomena. The Bayesian Abel bound is slightly higher than the BobrovskyZaka ï bound and, consequently, leads to a better prediction of the threshold effect with the same computational cost. The Weiss-Weinstein bounds obtained by numerical evaluation of (88) and (91) are the same; therefore, $\mathrm{s}=1 / 2$ seems to be the optimum value in this problem. As expected by the addition of constraints, the Weiss-Weinstein bounds provide a better prediction of the global MSE of the estimators in comparison with the Bobrovsky-Zakaï bound and the Bayesian Abel bound. The Weiss-Weinstein bound threshold value provides a better approximation of the effective SNR at which the estimators experience the threshold behavior.

\section{CONCLUSION}

In this paper, we proposed a framework to study the Bayesian minimal bounds on the MSE of the Weiss-Weinstein family. This framework is based on both the best Bayesian bound (MMSE) and a constrained optimization problem. By rewriting the problem of the MMSEE as a continuous constrained optimization problem and by relaxing these constraints, we reobtain the lower bounds of the Weiss-Weinstein family. Moreover, this framework allows us to propose new minimal bounds. In this way, we propose an extension of the Bayesian Bhattacharyya bound and a Bayesian version of the Abel bound. Additionally, we give some closed-form expressions of several minimal bounds for both a general Gaussian observation model with parameterized mean and a spectral analysis model.

\section{APPENDIX}

\section{A. Proof of Theorem 1}

This proof is based on the three following lemmas.

Lemma 1:

$$
\mathcal{C}_{1}=\mathcal{C}_{2}
$$

Lemma 2:

$$
\mathcal{C}_{1}=\mathcal{C}_{3}
$$

Lemma 3:

$$
\mathcal{C}_{1}=\mathcal{C}_{4}
$$

Proof of Lemma 1:

- $C_{2} \subset C_{1}$ : we assume that

$$
\begin{gathered}
\mathbf{S} \forall f(\mathbf{y}, \theta) \in \mathcal{F}, \iint_{\Theta} v(\mathbf{y}, \theta)(\partial f(\mathbf{y}, \theta) / \partial \theta) d \mathbf{y} d \theta= \\
\int_{\Theta} \int_{\Omega} f(\mathbf{y}, \theta) d \mathbf{y} d \theta . \mathrm{S}
\end{gathered}
$$

Since

$\frac{\partial[v(\mathbf{y}, \theta) f(\mathbf{y}, \theta)]}{\partial \theta}=v(\mathbf{y}, \theta) \frac{\partial f(\mathbf{y}, \theta)}{\partial \theta}+\frac{\partial v(\mathbf{y}, \theta)}{\partial \theta} f(\mathbf{y}, \theta)$

we have

$$
\begin{aligned}
& \int_{\Theta} \int_{\Omega} \frac{\partial[v(\mathbf{y}, \theta) f(\mathbf{y}, \theta)]}{\partial \theta}-\frac{\partial v(\mathbf{y}, \theta)}{\partial \theta} \\
& \quad \times f(\mathbf{y}, \theta) d \mathbf{y} d \theta=\int_{\Theta} \int_{\Omega} f(\mathbf{y}, \theta) d \mathbf{y} d \theta \\
& \Longrightarrow \int_{\Theta} \int_{\Omega}\left(1+\frac{\partial v(\mathbf{y}, \theta)}{\partial \theta}\right) f(\mathbf{y}, \theta) d \mathbf{y} d \theta=0 .
\end{aligned}
$$

Since (99) holds for any $f(\mathbf{y}, \theta)$, if we choose $f(\mathbf{y}, \theta)=$ $1+(\partial v(\mathbf{y}, \theta) / \partial \theta)$, we obtain

$$
\begin{gathered}
\int_{\Theta} \int_{\Omega}\left(1+\frac{\partial v(\mathbf{y}, \theta)}{\partial \theta}\right)^{2} d \mathbf{y} d \theta=0 \\
\quad \Longrightarrow 1+\frac{\partial v(\mathbf{y}, \theta)}{\partial \theta}=0 \\
\Longrightarrow v(\mathbf{y}, \theta)=z(\mathbf{y})-\theta
\end{gathered}
$$

where $z(\mathbf{y})$ is a function of $\mathbf{y}$ only.

- $C_{1} \subset C_{2}$ : on the other hand, if we assume that $v(\mathbf{y}, \theta)=$ $z(\mathbf{y})-\theta$, then

$$
\begin{aligned}
\int_{\Theta} & \int_{\Omega} v(\mathbf{y}, \theta) \frac{\partial f(\mathbf{y}, \theta)}{\partial \theta} d \mathbf{y} d \theta \\
= & \int_{\Theta} \int_{\Omega}(z(\mathbf{y})-\theta) \frac{\partial f(\mathbf{y}, \theta)}{\partial \theta} d \mathbf{y} d \theta \\
= & \int_{\Theta} \int_{\Omega} \frac{\partial[(z(\mathbf{y})-\theta) f(\mathbf{y}, \theta)]}{\partial \theta}+f(\mathbf{y}, \theta) d \mathbf{y} d \theta \\
= & \int_{\Theta} \int_{\Omega} f(\mathbf{y}, \theta) d \mathbf{y} d \theta \quad \forall f(\mathbf{y}, \theta) \in \mathcal{F} .
\end{aligned}
$$

These two items prove Lemma 1.

Proof of Lemma 2:

- $C_{3} \subset C_{1}$ : we assume that $\forall f(\mathbf{y}, \theta) \in \mathcal{F}$ such that $\int_{\Theta} \int_{\Omega} f(\mathbf{y}, \theta) d \mathbf{y} d \theta=1$ and $\forall h$ such that $\theta+h \in \Theta$

$\int_{\Theta} \int_{\Omega} v(\mathbf{y}, \theta)(f(\mathbf{y}, \theta+h)-f(\mathbf{y}, \theta)) d \mathbf{y} d \theta=h$.

Then, when $h \rightarrow 0$, we have

$\int_{\Theta} \int_{\Omega} v(\mathbf{y}, \theta) \frac{\partial f(\mathbf{y}, \theta)}{\partial \theta} d \mathbf{y} d \theta=1 \Longrightarrow v(\mathbf{y}, \theta)=z(\mathbf{y})-\theta$

thanks to the result of the first item of Lemma 1. 
- $C_{1} \subset C_{3}$ : on the other hand, if we assume $v(\mathbf{y}, \theta)=$ $z(\mathbf{y})-\theta$, then by setting $\varphi=\theta+h$

$$
\begin{aligned}
\int_{\Theta} & \int_{\Omega} v(\mathbf{y}, \theta) f(\mathbf{y}, \theta+h) d \mathbf{y} d \theta \\
= & \int_{\Theta} \int_{\Omega}(z(\mathbf{y})-\theta) f(\mathbf{y}, \theta+h) d \mathbf{y} d \theta \\
& =\int_{\Theta} \int_{\Omega}(z(\mathbf{y})-\varphi+h) f(\mathbf{y}, \varphi) d \mathbf{y} d \varphi \\
& =\int_{\Theta} \int_{\Omega}(z(\mathbf{y})-\varphi) f(\mathbf{y}, \varphi) d \mathbf{y} d \varphi+h
\end{aligned}
$$

leading to

$$
\int_{\Theta} \int_{\Omega}(z(\mathbf{y})-\theta)(f(\mathbf{y}, \theta+h)-f(\mathbf{y}, \theta)) d \mathbf{y} d \theta=h
$$

$\forall f(\mathbf{y}, \theta) \in \mathcal{F}$ such that $\iint_{\Omega} f(\mathbf{y}, \theta) d \mathbf{y} d \theta=1$ and $\forall h$ such that $\theta+h \in \Theta$.

These two items prove Lemma 2.

\section{Proof of Lemma 3:}

- $C_{4} \subset C_{1}$ : let $L(\mathbf{y}, \eta, \theta)=(f(\mathbf{y}, \eta) / f(\mathbf{y}, \theta))$ and assume that $\forall f(\mathbf{y}, \theta) \in \mathcal{F}$ such that $\int_{\Theta} \int_{\Omega} f(\mathbf{y}, \theta) d \mathbf{y} d \theta=1, \forall h$ such that $\theta \pm h \in \Theta$ and $\forall s \in[0,1]$

$$
\begin{aligned}
& \iint_{\Theta} v(\mathbf{y}, \theta)\left[L^{s}(\mathbf{y}, \theta+h, \theta)\right. \\
& \left.-L^{1-s}(\mathbf{y}, \theta-h, \theta)\right] f(\mathbf{y}, \theta) d \mathbf{y} d \theta \\
& \quad=h \int_{\Theta} \int_{\Omega} L^{1-s}(\mathbf{y}, \theta-h, \theta) f(\mathbf{y}, \theta) d \mathbf{y} d \theta .
\end{aligned}
$$

Then, when $s \rightarrow 1$, we obtain

$$
\begin{aligned}
\int_{\Theta} \int_{\Omega} v(\mathbf{y}, \theta)(f(\mathbf{y}, \theta+h)- & f(\mathbf{y}, \theta)) d \mathbf{y} d \theta=h \\
\Longrightarrow v(\mathbf{y}, \theta) & =z(\mathbf{y})-\theta
\end{aligned}
$$

thanks to the result of the first item of Lemma 2.

- $C_{1} \subset C_{4}$ : on the other hand, if we assume $v(\mathbf{y}, \theta)=$ $z(\mathbf{y})-\theta$, then by letting $\varphi=\theta+h$

$$
\begin{aligned}
\int_{\Theta} \int_{\Omega} v(\mathbf{y}, \theta) L^{s}(\mathbf{y}, \theta+h, \theta) f(\mathbf{y}, \theta) d \theta d \mathbf{y} \\
=\int_{\Theta} \int_{\Omega}(z(\mathbf{y})-\theta) L^{s}(\mathbf{y}, \theta+h, \theta) f(\mathbf{y}, \theta) d \theta d \mathbf{y} \\
=\int_{\Theta} \int_{\Omega}(z(\mathbf{y})-\varphi) L^{1-s}(\mathbf{y}, \varphi-h, \varphi) f(\mathbf{y}, \varphi) d \varphi d \mathbf{y} \\
\quad+h \int_{\Theta} \int_{\Omega} L^{1-s}(\mathbf{y}, \varphi-h, \varphi) f(\mathbf{y}, \varphi) d \varphi d \mathbf{y}
\end{aligned}
$$

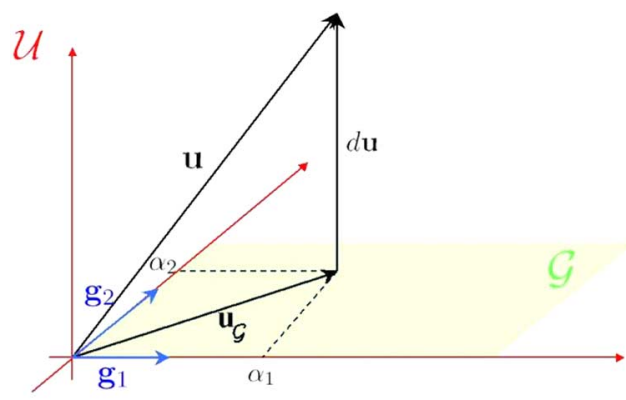

Fig. 2. Graphical representation of the problem.

leading to

$$
\begin{aligned}
& \int_{\Theta} \int_{\Omega} v(\mathbf{y}, \theta)\left[L^{s}(\mathbf{y}, \theta+h, \theta)\right. \\
& \left.-L^{1-s}(\mathbf{y}, \theta-h, \theta)\right] f(\mathbf{y}, \theta) d \theta d \mathbf{y} \\
& \quad=h \int_{\Theta} \int_{\Omega} L^{1-s}(\mathbf{y}, \theta-h, \theta) f(\mathbf{y}, \theta) d \theta d \mathbf{y}
\end{aligned}
$$

$\forall f(\mathbf{y}, \theta) \in \mathcal{F}$ such that $\iint_{\Theta} f(\mathbf{y}, \theta) d \mathbf{y} d \theta=1, \forall h$ such that $\theta \pm h \in \Theta$ and $\forall s \in[0,1]$.

These two items prove Lemma 3.

Lemmas 1, 2, and 3 prove Theorem 1.

\section{B. Proof of Theorem 2}

Let $\mathcal{U}$ be a vector space of any dimension on the field of real numbers $\mathbb{R}$, with an inner product denoted by $\langle\mathbf{u}, \mathbf{w}\rangle$, where $\mathbf{u}$ and $\mathbf{w}$ are two vectors of $\mathcal{U}$. Let $\left\{\mathbf{g}_{1}, \ldots, \mathbf{g}_{K}\right\}$ be a family of $K$ independent vectors of $\mathcal{U}$ and $\mathbf{c}=\left[\begin{array}{lll}c_{1} & \cdots & c_{K}\end{array}\right]^{T}$ be a vector of $\mathbb{R}^{K}$. We are interested in the solution of the minimization of $\langle\mathbf{u}, \mathbf{u}\rangle$ subject to the following $K$ linear constraints $\left\langle\mathbf{u}, \mathbf{g}_{k}\right\rangle=$ $c_{k}, k \in[1, K]$.

Let $\mathcal{G}$ be the vectorial subspace of dimension $K$ generated by the elements $\left\{\mathbf{g}_{1}, \ldots, \mathbf{g}_{K}\right\}$. Then, $\forall \mathbf{u} \in \mathcal{U}, \mathbf{u}=\mathbf{u}_{\mathcal{G}}+d \mathbf{u}$, where $\mathbf{u}_{\mathcal{G}}$ is the orthogonal projection of $\mathbf{u}$ on $\mathcal{G}$, i.e., the vector $\mathbf{u}_{\mathcal{G}} \in \mathcal{G}$ such that $\left\langle\mathbf{u}-\mathbf{u}_{\mathcal{G}}, \mathbf{g}_{k}\right\rangle=0, k \in[1, K]$ (see Fig. 2) for a graphical representation).

Let $\boldsymbol{\alpha}=\left[\begin{array}{lll}\alpha_{1} & \cdots & \alpha_{K}\end{array}\right]^{T}$ be the coordinates of $\mathbf{u}_{\mathcal{G}}$ in the basis $\left\{\mathbf{g}_{1}, \ldots, \mathbf{g}_{K}\right\}$ of $\mathcal{G}$ (i.e., $\mathbf{u}_{\mathcal{G}}=\sum_{k=1}^{K} \alpha_{k} \mathbf{g}_{k}$ ). These coordinates satisfy: $\left\langle\mathbf{u}, \mathbf{g}_{k}\right\rangle=\left\langle\mathbf{u}_{\mathcal{G}}, \mathbf{g}_{k}\right\rangle, k \in[1, K]$. Moreover, if $\mathbf{u}$ satisfies the $K$ constraints $\left\langle\mathbf{u}, \mathbf{g}_{k}\right\rangle=c_{k}, k \in[1, K]$, then

$$
\begin{aligned}
\left\langle\mathbf{u}, \mathbf{g}_{k}\right\rangle & =c_{k} \\
& \Rightarrow\left\langle\mathbf{u}_{\mathcal{G}}, \mathbf{g}_{k}\right\rangle=c_{k} \\
& \Rightarrow\left\langle\sum_{l=1}^{K} \alpha_{l} \mathbf{g}_{l}, \mathbf{g}_{k}\right\rangle=c_{k} \\
& \Rightarrow \sum_{l=1}^{K} \alpha_{l}\left\langle\mathbf{g}_{l}, \mathbf{g}_{k}\right\rangle=c_{k}
\end{aligned}
$$

i.e., by a matricial rewriting $\mathbf{G} \boldsymbol{\alpha}=\mathbf{c}$, where $\mathbf{G}$ is the Gram matrix associated to the family $\left\{\mathbf{g}_{1}, \ldots, \mathbf{g}_{K}\right\}: G_{k, l}=\left\langle\mathbf{g}_{l}, \mathbf{g}_{k}\right\rangle$. The equation $\mathbf{G} \boldsymbol{\alpha}=\mathbf{c}$ has for unique solution $\boldsymbol{\alpha}=\mathbf{G}^{-1} \mathbf{c}$. Let 
$\mathbf{u}_{\mathcal{G}, \mathbf{c}}$ be the vector of $\mathcal{G}$ corresponding to this solution. Then, $\forall \mathbf{u} \in \mathcal{U}$ and for satisfying the $K$ aforementioned constraints we have $\langle\mathbf{u}, \mathbf{u}\rangle=\left\langle\mathbf{u}_{\mathcal{G}, \mathbf{c}}, \mathbf{u}_{\mathcal{G}, \mathbf{c}}\right\rangle+\langle d \mathbf{u}, d \mathbf{u}\rangle \geq\left\langle\mathbf{u}_{\mathcal{G}, \mathbf{c}}, \mathbf{u}_{\mathcal{G}, \mathbf{c}}\right\rangle$, and the minimum is achieved for $d \mathbf{u}=0$, which means that $\mathbf{u}_{\mathcal{G}, \mathbf{c}}$ is the solution of the problem. The value of the minimal norm is given by

$$
\begin{aligned}
\left\langle\mathbf{u}_{\mathcal{G}, \mathbf{c}}, \mathbf{u}_{\mathcal{G}, \mathbf{c}}\right\rangle & =\left\langle\sum_{k=1}^{K} \alpha_{k} \mathbf{g}_{k}, \sum_{l=1}^{K} \alpha_{l} \mathbf{g}_{l}\right\rangle \\
& =\sum_{k=1}^{K} \sum_{l=1}^{K} \alpha_{k} \alpha_{l}\left\langle\mathbf{g}_{k}, \mathbf{g}_{l}\right\rangle \\
& =\boldsymbol{\alpha}^{T} \mathbf{G} \boldsymbol{\alpha} \\
& =\left(\mathbf{G}^{-1} \mathbf{c}\right)^{T} \mathbf{G} \mathbf{G}^{-1} \mathbf{c} \\
& =\mathbf{c}^{T} \mathbf{G}^{-1} \mathbf{c} .
\end{aligned}
$$

\section{Derivation of the Bayesian Abel Bound}

We have to calculate the quadratic form $\mathbf{c}^{T} \mathbf{G}^{-1} \mathbf{c}$ (29). Since

$$
\int_{\Theta} \int_{\Omega} p\left(\mathbf{x}, \theta+h_{i}\right) d \theta d \mathbf{x}=1 \quad \forall h_{i} \in \mathbb{R}
$$

and, due to (9)

$$
\int_{\Theta} \int_{\Omega} \frac{\partial^{i} p(\mathbf{x}, \theta)}{\partial \theta^{i}} d \theta d \mathbf{x}=0 \quad \forall i \geq 1
$$

the matrix $\mathbf{G}=\iint_{\Theta} \operatorname{gg}^{T} d \theta d \mathbf{x}$ can now be written as the following partitioned matrix:

$$
\mathbf{G}=\left(\begin{array}{ccc}
1 & \mathbf{0}_{1 \times m} & \mathbf{0}_{1 \times r} \\
\mathbf{0}_{m \times 1} & \mathbf{B} & \boldsymbol{\Gamma}^{T} \\
\mathbf{0}_{r \times 1} & \boldsymbol{\Gamma} & \mathbf{D}
\end{array}\right)
$$

where the elements $\{\mathbf{B}\}_{i, j}$ and $\{\mathbf{D}\}_{i, j}$ of the matrices $\mathbf{B}(m \times$ $m)$ and $\mathbf{D}(r \times r)$ are given by (37) and (48), respectively, and the element $\{\boldsymbol{\Gamma}\}_{i, j}$ of the matrix $\boldsymbol{\Gamma}(r \times m)$ is given by

$$
\begin{aligned}
\left\{\boldsymbol{\Gamma}_{i, j}\right. & =\int_{\Theta} \int_{\Omega} \frac{p\left(\mathbf{x}, \theta+h_{i}\right)-p(\mathbf{x}, \theta)}{p(\mathbf{x}, \theta)} \frac{\partial^{j} p(\mathbf{x}, \theta)}{\partial \theta^{j}} d \theta d \mathbf{x} \\
& =\int_{\Theta} \int_{\Omega} \frac{p\left(\mathbf{x}, \theta+h_{i}\right)}{p(\mathbf{x}, \theta)} \frac{\partial^{j} p(\mathbf{x}, \theta)}{\partial \theta^{j}} d \theta d \mathbf{x} .
\end{aligned}
$$

Let $\tilde{\mathbf{G}}=\left(\begin{array}{cc}\mathbf{B} & \boldsymbol{\Gamma}^{T} \\ \boldsymbol{\Gamma} & \mathbf{D}\end{array}\right)$ and $\mathbf{c}=\left[\begin{array}{lll}0 & \boldsymbol{\alpha}^{T} & \mathbf{h}^{T}\end{array}\right]^{T}$, where $\boldsymbol{\alpha}=$ $\left[\begin{array}{llll}1 & 0 & \cdots & 0\end{array}\right]^{T}($ size $m \times 1)$, and $\mathbf{h}=\left[\begin{array}{lll}h_{1} & \cdots & h_{r}\end{array}\right]^{T}$. Since the first element of $\mathbf{c}$ is null, only the right bottom corner $\tilde{\mathbf{G}}^{-1}(\operatorname{size}(m+r) \times(m+r))$ of $\mathbf{G}^{-1}$ is of interest. $\tilde{\mathbf{G}}^{-1}$ is given straightforwardly by

$$
\tilde{\mathbf{G}}^{-1}=\left(\begin{array}{cc}
\mathbf{B} & \boldsymbol{\Gamma}^{T} \\
\mathbf{\Gamma} & \mathbf{D}
\end{array}\right)^{-1}
$$

Consequently, the Bayesian Abel bound denoted $\mathrm{BAB}_{m, r}$ is then given by

$$
\mathrm{BAB}_{m, r}=\left[\begin{array}{ll}
\boldsymbol{\alpha}^{T} & \mathbf{h}^{T}
\end{array}\right]\left(\begin{array}{cc}
\mathbf{B} & \boldsymbol{\Gamma}^{T} \\
\boldsymbol{\Gamma} & \mathbf{D}
\end{array}\right)^{-1}\left[\begin{array}{l}
\boldsymbol{\alpha} \\
\mathbf{h}
\end{array}\right] .
$$

After some algebraic effort, we obtain the final form

$$
\mathrm{BAB}_{m, r}=\boldsymbol{\alpha}^{T} \mathbf{B}^{-1} \boldsymbol{\alpha}+\mathbf{u}^{T} \mathbf{J}^{-1} \mathbf{u}
$$

with

$$
\left\{\begin{array}{l}
\mathbf{u}=\Gamma \mathbf{B}^{-1} \boldsymbol{\alpha}-\mathbf{h} \\
\mathbf{J}=\mathbf{D}-\Gamma \mathbf{B}^{-1} \Gamma^{T}
\end{array}\right.
$$

\section{Minimal Bounds Derivation for the Gaussian Observation Model With Parameterized Mean}

1) Bayesian Cramér-Rao Bound: The Bayesian CramérRao bound can be divided into two terms [20]

$\mathrm{BCRB}$

$$
=\left(\int_{\Theta} \operatorname{CRB}^{-1}(\theta) p(\theta) d \theta-\int_{\Theta} \frac{\partial^{2} \ln p(\theta)}{\partial \theta^{2}} p(\theta) d \theta\right)^{-1}
$$

where $\operatorname{CRB}(\theta)$ is the standard (i.e., deterministic) Cramér-Rao bound given by [68]:

$$
\operatorname{CRB}\left(\theta_{0}\right)=\frac{\sigma^{2}}{2\left\|\left.\frac{\partial \mathbf{m}(\theta)}{\partial \theta}\right|_{\theta_{0}}\right\|^{2}}
$$

where $\theta_{0}$ is the true value of the parameter in the deterministic context.

The second term of (120) is

$$
\begin{aligned}
\int_{\Theta} & \frac{\partial^{2} \ln p(\theta)}{\partial \theta^{2}} p(\theta) d \theta \\
= & -\frac{1}{2 \sigma_{\theta}^{2}} \int_{\Theta} \frac{\partial^{2}(\theta-\mu)^{2}}{\partial \theta^{2}} p(\theta) d \theta \\
= & -\frac{1}{\sigma_{\theta}^{2}} \int_{\Theta} p(\theta) d \theta=-\frac{1}{\sigma_{\theta}^{2}} .
\end{aligned}
$$

Consequently

$$
\mathrm{BCRB}=\frac{\sigma_{\theta}^{2}}{\frac{2 \sigma_{\theta}^{2}}{\sigma^{2}} \mathbb{E}_{\theta}\left[\left\|\frac{\partial \mathbf{m}(\theta)}{\partial \theta}\right\|^{2}\right]+1}
$$

2) Bobrovsky-Zakaï Bound: The Bobrovsky-Zakaï bound is given by

$$
\mathrm{BZB}=\sup _{h} \frac{h^{2}}{\iint_{\Omega} \frac{p^{2}(\mathbf{y}, \theta+h)}{p(\mathbf{y}, \theta)} d \mathbf{y} d \theta-1} .
$$


The double integral in the last equation can be rewritten as follows:

$$
\begin{aligned}
& \int_{\Theta} \int_{\Omega} \frac{p^{2}(\mathbf{y}, \theta+h)}{p(\mathbf{y}, \theta)} d \mathbf{y} d \theta \\
&=\int_{\Theta} \frac{p^{2}(\theta+h)}{p(\theta)} \int_{\Omega} \frac{p^{2}(\mathbf{y} \mid \theta+h)}{p(\mathbf{y} \mid \theta)} d \mathbf{y} d \theta .
\end{aligned}
$$

The term $\left(p^{2}(\mathbf{y} \mid \theta+h) / p(\mathbf{y} \mid \theta)\right)$ becomes (126), shown at the bottom of the page.

Let $\mathbf{x}=\mathbf{y}-2 \mathbf{m}(\theta+h)+\mathbf{m}(\theta)$, and note that

$$
\begin{aligned}
\|\mathbf{x}\|^{2}=\|\mathbf{y}\|^{2}+\| 2 \mathbf{m}(\theta+h) & -\mathbf{m}(\theta) \|^{2} \\
& -2 \operatorname{Re}\left\{\mathbf{y}^{H}(2 \mathbf{m}(\theta+h)-\mathbf{m}(\theta))\right\} .
\end{aligned}
$$

Consequently [see (127) at the bottom of the page].

The Bobrovsky-Zakaï bound is finally given by

$$
\mathrm{BZB}=\sup _{h} \frac{h^{2}}{\int_{\Theta} \frac{p^{2}(\theta+h)}{p(\theta)} e^{\frac{2}{\sigma^{2}}\|\mathbf{m}(\theta+h)-\mathbf{m}(\theta)\|^{2}} d \theta-1} .
$$

3) Bayesian Abel Bound: We have to calculate

$$
\begin{aligned}
\phi(h) & =\frac{1}{h} \int_{\Theta} \int_{\Omega} \frac{\partial \ln p(\mathbf{y}, \theta)}{\partial \theta} p(\mathbf{y}, \theta+h) d \mathbf{y} d \theta \\
& =\frac{1}{h} \int_{\Theta} p(\theta+h) \int_{\Omega}\left(\frac{\partial \ln p(\mathbf{y} \mid \theta)+\ln p(\theta)}{\partial \theta}\right)
\end{aligned}
$$

$$
\begin{aligned}
& \times p(\mathbf{y} \mid \theta+h) d \mathbf{y} d \theta \\
= & \frac{1}{h} \int_{\Theta} p(\theta+h) \int_{\Omega} \frac{\partial \ln p(\mathbf{y} \mid \theta)}{\partial \theta} p(\mathbf{y} \mid \theta+h) d \mathbf{y} d \theta \\
& +\frac{1}{h} \int_{\Theta} \frac{\partial \ln p(\theta)}{\partial \theta} p(\theta+h) d \theta
\end{aligned}
$$

The first term in (129) is given by

$$
\begin{aligned}
\int_{\Omega} & \frac{\partial \ln p(\mathbf{y} \mid \theta)}{\partial \theta} p(\mathbf{y} \mid \theta+h) d \mathbf{y} \\
= & -\frac{1}{\sigma^{2}} \int_{\Omega} \frac{\partial(\mathbf{y}-\mathbf{m}(\theta))^{H}(\mathbf{y}-\mathbf{m}(\theta))}{\partial \theta} p(\mathbf{y} \mid \theta+h) d \mathbf{y} \\
= & \frac{2}{\sigma^{2}} \int_{\Omega} \operatorname{Re}\left\{\frac{\partial \mathbf{m}^{H}(\theta)}{\partial \theta}(\mathbf{y}-\mathbf{m}(\theta))\right\} p(\mathbf{y} \mid \theta+h) d \mathbf{y} \\
= & \frac{2}{\sigma^{2}} \operatorname{Re}\left\{\frac{\partial \mathbf{m}^{H}(\theta)}{\partial \theta}\left(\int_{\Omega} \mathbf{y} p(\mathbf{y} \mid \theta+h) d \mathbf{y}-\mathbf{m}(\theta)\right)\right\} \\
= & \frac{2}{\sigma^{2}} \operatorname{Re}\left\{\frac{\partial \mathbf{m}^{H}(\theta)}{\partial \theta}(\mathbf{m}(\theta+h)-\mathbf{m}(\theta))\right\} .
\end{aligned}
$$

For the second term in (129), we have

$$
\begin{aligned}
& \frac{1}{h} \int_{\Theta} p(\theta+h) \frac{\partial \ln p(\theta)}{\partial \theta} d \theta \\
& \quad=-\frac{1}{h \sigma_{\theta}^{2}} \int_{\Theta}(\theta-\mu) p(\theta+h) d \theta \\
& \quad=\frac{1}{\sigma_{\theta}^{2}} .
\end{aligned}
$$

$$
\begin{aligned}
& \frac{p^{2}(\mathbf{y} \mid \theta+h)}{p(\mathbf{y} \mid \theta)} \\
& \quad=\frac{1}{\left(\pi \sigma^{2}\right)^{N}} e^{-\frac{1}{\sigma^{2}}\left(2(\mathbf{y}-\mathbf{m}(\theta+h))^{H}(\mathbf{y}-\mathbf{m}(\theta+h))-(\mathbf{y}-\mathbf{m}(\theta))^{H}(\mathbf{y}-\mathbf{m}(\theta))\right)} \\
& \quad=\frac{1}{\left(\pi \sigma^{2}\right)^{N}} e^{-\frac{1}{\sigma^{2}}\left(\|\mathbf{y}\|^{2}+2\|\mathbf{m}(\theta+h)\|^{2}-\|\mathbf{m}(\theta)\|^{2}-2 \operatorname{Re}\left\{\mathbf{y}^{H}(2 \mathbf{m}(\theta+h)-\mathbf{m}(\theta))\right\}\right)} .
\end{aligned}
$$

$$
\begin{aligned}
& \int_{\Omega} \frac{p^{2}(\mathbf{y} \mid \theta+h)}{p(\mathbf{y} \mid \theta)} d \mathbf{y} \\
& =\frac{1}{\left(\pi \sigma^{2}\right)^{N}} \int_{\Omega} e^{-\frac{1}{\sigma^{2}}\left(\|\mathbf{x}\|^{2}+2\|\mathbf{m}(\theta+h)\|^{2}-\|\mathbf{m}(\theta)\|^{2}-\|2 \mathbf{m}(\theta+h)-\mathbf{m}(\theta)\|^{2}\right)} d \mathbf{x} \\
& =\frac{1}{\left(\pi \sigma^{2}\right)^{N}} e^{-\frac{1}{\sigma^{2}}\left(2\|\mathbf{m}(\theta+h)\|^{2}+\|\mathbf{m}(\theta)\|^{2}-\|2 \mathbf{m}(\theta+h)-\mathbf{m}(\theta)\|^{2}\right)} \underbrace{\int_{\Omega} e^{-\frac{1}{\sigma^{2}}\|\mathbf{x}\|^{2}} d \mathbf{x}}_{=\left(\pi \sigma^{2}\right)^{N}} \\
& =e^{\frac{2}{\sigma^{2}}\|\mathbf{m}(\theta+h)-\mathbf{m}(\theta)\|^{2}} .
\end{aligned}
$$


Finally

$$
\begin{aligned}
\phi(h) & =\frac{1}{\sigma_{\theta}^{2}}+\frac{2}{h \sigma^{2}} \\
& \times \mathbb{E}_{\theta+h}\left[\operatorname{Re}\left\{\frac{\partial \mathbf{m}^{H}(\theta)}{\partial \theta}(\mathbf{m}(\theta+h)-\mathbf{m}(\theta))\right\}\right] .
\end{aligned}
$$

4) Weiss-Weinstein Bound: We have to calculate

$$
\eta(\alpha, \beta)=\ln \int_{\Theta} \int_{\Omega} \frac{p^{\alpha}(\mathbf{y}, \theta+\beta)}{p^{\alpha-1}(\mathbf{y}, \theta)} d \mathbf{y} d \theta .
$$

This function can be modified as follows:

$$
\eta(\alpha, \beta)=\ln \int_{\Theta} \frac{p^{\alpha}(\theta+\beta)}{p^{\alpha-1}(\theta)} \int_{\Omega} \frac{p^{\alpha}(\mathbf{y} \mid \theta+\beta)}{p^{\alpha-1}(\mathbf{y} \mid \theta)} d \mathbf{y} d \theta .
$$

Let us first study the term shown in (135) at the bottom of the page.

Let $\mathbf{x}=\mathbf{y}-(\alpha \mathbf{m}(\theta+\beta)-(\alpha-1) \mathbf{m}(\theta))$. Note that

$$
\begin{aligned}
\|\mathbf{x}\|^{2}=\|\mathbf{y}\|^{2}+ & \|\alpha \mathbf{m}(\theta+\beta)-(\alpha-1) \mathbf{m}(\theta)\|^{2} \\
& -2 \operatorname{Re}\left\{\mathbf{y}^{H}(\alpha \mathbf{m}(\theta+\beta)-(\alpha-1) \mathbf{m}(\theta))\right\} .
\end{aligned}
$$

Consequently, [see (137) at the bottom of the page].

For the second term

$$
\frac{p^{\alpha}(\theta+\beta)}{p^{\alpha-1}(\theta)}=\frac{1}{\sqrt{2 \pi} \sigma_{\theta}} e^{-\frac{1}{2 \sigma_{\theta}^{2}}\left[\alpha(\theta+\beta-\mu)^{2}-(\alpha-1)(\theta-\mu)^{2}\right]} .
$$

Finally, the semi-invariant moment generating function is given by (139), shown at the bottom of the page.

\section{E. Bayesian Bounds Derivation for a Spectral Analysis Problem}

1) Cramér-Rao Bound: The Bayesian Cramér-Rao bound is given by (123)

$$
\mathrm{BCRB}=\frac{\sigma_{\theta}^{2}}{\frac{2 \sigma_{\theta}^{2}}{\sigma^{2}} \mathbb{E}_{\theta}\left[\left\|\frac{\partial \mathbf{m}(\theta)}{\partial \theta}\right\|^{2}\right]+1}
$$

The term $\|(\partial \mathbf{m}(\theta) / \partial \theta)\|^{2}$ can be written

$$
\begin{aligned}
\left\|\frac{\partial \mathbf{m}(\theta)}{\partial \theta}\right\|^{2} & =\left\|a \frac{\partial \mathbf{s}(\theta)}{\partial \theta}\right\|^{2} \\
& =\sum_{k=0}^{N-1} a^{2}\left(j 2 \pi k e^{j 2 \pi k \theta}\right)\left(-j 2 \pi k e^{-j 2 \pi k \theta}\right) \\
& =a^{2} 4 \pi^{2} \sum_{k=0}^{N-1} k^{2}=\frac{2(a \pi)^{2}}{3} N(2 N-1)(N-1)
\end{aligned}
$$

which is independent of $\theta$. Consequently, the Bayesian Cramér-Rao bound is

$$
\mathrm{BCRB}=\frac{\sigma_{\theta}^{2}}{\operatorname{SNR} \frac{4 \pi^{2} \sigma_{\theta}^{2}}{3} N(2 N-1)(N-1)+1} .
$$

$$
\begin{aligned}
& \frac{p^{\alpha}(\mathbf{y} \mid \theta+\beta)}{p^{\alpha-1}(\mathbf{y} \mid \theta)} \\
& =\frac{1}{\left(\pi \sigma^{2}\right)^{N}} e^{-\frac{1}{\sigma^{2}}\left(\alpha(\mathbf{y}-\mathbf{m}(\theta+\beta))^{H}(\mathbf{y}-\mathbf{m}(\theta+\beta))-(\alpha-1)(\mathbf{y}-\mathbf{m}(\theta))^{H}(\mathbf{y}-\mathbf{m}(\theta))\right)} \\
& =\frac{1}{\left(\pi \sigma^{2}\right)^{N}} e^{-\frac{1}{\sigma^{2}}\left(\|\mathbf{y}\|^{2}+\alpha\|\mathbf{m}(\theta+\beta)\|^{2}-(\alpha-1)\|\mathbf{m}(\theta)\|^{2}-2 \operatorname{Re}\left\{\mathbf{y}^{H}(\alpha \mathbf{m}(\theta+\beta)-(\alpha-1) \mathbf{m}(\theta))\right\}\right) .}
\end{aligned}
$$

$$
\begin{aligned}
& \int_{\Omega} \frac{p^{\alpha}(\mathbf{y} \mid \theta+\beta)}{p^{\alpha-1}(\mathbf{y} \mid \theta)} d \mathbf{y} \\
& =\frac{1}{\left(\pi \sigma^{2}\right)^{N}} \int_{\Omega} e^{-\frac{1}{\sigma^{2}}\left(\|\mathbf{x}\|^{2}-\|\alpha \mathbf{m}(\theta+\beta)-(\alpha-1) \mathbf{m}(\theta)\|^{2}+\alpha\|\mathbf{m}(\theta+\beta)\|^{2}-(\alpha-1)\|\mathbf{m}(\theta)\|^{2}\right)} d \mathbf{x} \\
& =\frac{1}{\left(\pi \sigma^{2}\right)^{N}} e^{-\frac{1}{\sigma^{2}}\left(-\|\alpha \mathbf{m}(\theta+\beta)-(\alpha-1) \mathbf{m}(\theta)\|^{2}+\alpha\|\mathbf{m}(\theta+\beta)\|^{2}-(\alpha-1)\|\mathbf{m}(\theta)\|^{2}\right)} \underbrace{\int_{\Omega} e^{-\frac{\|x\|^{2}}{\sigma^{2}} d \mathbf{x}}}_{=\left(\pi \sigma^{2}\right)^{N}} \\
& =e^{\frac{\alpha(\alpha-1)}{\sigma^{2}}\|\mathbf{m}(\theta+\beta)-\mathbf{m}(\theta)\|^{2}} .
\end{aligned}
$$

$$
\eta(\alpha, \beta)=\ln \frac{1}{\sqrt{2 \pi} \sigma_{\theta}} \int_{\Theta} e^{\frac{\alpha(\alpha-1)}{\sigma^{2}}\|\mathbf{m}(\theta+\beta)-\mathbf{m}(\theta)\|^{2}-\frac{1}{2 \sigma_{\theta}^{2}}(\theta-(\sqrt{\alpha(\alpha-1)}-\alpha) h-\mu)(\theta+(\sqrt{\alpha(\alpha-1)}+\alpha) h-\mu)} d \theta
$$


2) Bobrovsky-Zakaï Bound: The Bobrovsky-Zakaï bound is given by (128)

$$
\mathrm{BZB}=\sup _{h} \frac{h^{2}}{\int_{\Theta} \frac{p^{2}(\theta+h)}{p(\theta)} e^{\frac{2}{\sigma^{2}}\|\mathbf{m}(\theta+h)-\mathbf{m}(\theta)\|^{2}} d \theta-1} .
$$

In the case of our specific model (79), the term $\| \mathbf{m}(\theta+h)-$ $\mathbf{m}(\theta) \|^{2}$ can be written

$$
\begin{aligned}
& \|\mathbf{m}(\theta+h)-\mathbf{m}(\theta)\|^{2} \\
& =a^{2} \sum_{k=0}^{N-1}\left(e^{j 2 \pi k(\theta+h)}-e^{j 2 \pi k \theta}\right)\left(e^{-j 2 \pi k(\theta+h)}-e^{-j 2 \pi k \theta}\right) \\
& =a^{2} \sum_{k=0}^{N-1}\left(2-2 \operatorname{Re}\left\{e^{j 2 \pi k h}\right\}\right)=2 a^{2} \sum_{k=0}^{N-1} 1-\cos (2 \pi k h) \\
& =2 a^{2}\left(N-\sin ^{2}(\pi h N)-\frac{1}{2} \frac{\sin (2 \pi h N)}{\tan (\pi h)}\right)
\end{aligned}
$$

which is independent of $\theta$. The term $\int_{\Theta}\left(p^{2}(\theta+h) / p(\theta)\right) d \theta$ becomes

$$
\begin{aligned}
& \int_{\Theta} \frac{p^{2}(\theta+h)}{p(\theta)} d \theta \\
& =\frac{1}{\sqrt{2 \pi} \sigma_{\theta}} e^{-\frac{1}{2 \sigma_{\theta}^{2}}\left[2 h^{2}-4 h \mu+\mu^{2}\right]} \int_{\Theta} e^{-\frac{1}{2 \sigma_{\theta}^{2}}\left[\theta^{2}+2 \theta(2 h-\mu)\right]} d \theta \\
& =e^{-\frac{1}{2 \sigma_{\theta}^{2}}\left[2 h^{2}-4 h \mu+\mu^{2}\right]+\frac{(2 h-\mu)^{2}}{2 \sigma_{\theta}^{2}}}=e^{\frac{h^{2}}{\sigma_{\theta}^{2}}}
\end{aligned}
$$

where the term $\int e^{-\left(1 / 2 \sigma_{\theta}^{2}\right)\left[\theta^{2}+2 \theta(2 h-\mu)\right]} d \theta$ is given by [71, $\mathrm{p}$. 355 , eq. (BI (28) (1))]

$$
\int_{-\infty}^{\infty} e^{-p^{2} x^{2} \pm q x} d x=\frac{\sqrt{\pi}}{\operatorname{abs}(p)} e^{\frac{q^{2}}{4 p^{2}}} .
$$

Finally, the Bobrovsky-Zakaï is given by

$$
\mathrm{BZB}=\sup _{h} \frac{h^{2}}{e^{4 \mathrm{SNR}\left(N-\sin ^{2}(\pi h N)-\frac{1}{2} \frac{\sin (2 \pi h N)}{\tan (\pi h)}\right)+\frac{h^{2}}{\sigma_{\theta}^{2}}}-1} .
$$

3) Bayesian Abel Bound: We have to calculate (132)

$$
\begin{aligned}
\phi(h)= & \frac{1}{\sigma_{\theta}^{2}}+\frac{2}{h \sigma^{2}} \\
& \mathbb{E}_{\theta+h}\left[\operatorname{Re}\left\{\frac{\partial \mathbf{m}^{H}(\theta)}{\partial \theta}(\mathbf{m}(\theta+h)-\mathbf{m}(\theta))\right\}\right] .
\end{aligned}
$$

The term $\operatorname{Re}\left\{\left(\partial \mathbf{m}^{H}(\theta) / \partial \theta\right)(\mathbf{m}(\theta+h)-\mathbf{m}(\theta))\right\}$ can be rewritten as follows:

$$
\begin{aligned}
\operatorname{Re} & \left\{\frac{\partial \mathbf{m}^{H}(\theta)}{\partial \theta}(\mathbf{m}(\theta+h)-\mathbf{m}(\theta))\right\} \\
= & \operatorname{Re}\left\{a^{2} \frac{\partial \mathbf{s}^{H}(\theta)}{\partial \theta}(\mathbf{s}(\theta+h)-\mathbf{s}(\theta))\right\} \\
= & a^{2} \operatorname{Re}\left\{\sum_{k=0}^{N-1}\left(e^{j 2 \pi k(\theta+h)}-e^{j 2 \pi k \theta}\right) \frac{\partial e^{-j 2 \pi k \theta}}{\partial \theta}\right\}
\end{aligned}
$$

$$
\begin{aligned}
= & -2 \pi a^{2} \operatorname{Re}\left\{\sum_{k=0}^{N-1} j k\left(e^{j 2 \pi k h}-1\right)\right\} \\
= & 2 \pi a^{2} \sum_{k=0}^{N-1} k \sin (2 \pi k h) \\
= & \pi a^{2}\left(N \frac{\cos (2 \pi h N)}{\tan (\pi h)}\right. \\
& \left.-\sin (2 \pi h N)\left(\frac{1}{2 \sin (\pi h)}+N\right)\right)
\end{aligned}
$$

which is independent of $\theta$. Consequently

$$
\begin{aligned}
\phi(h)=\frac{1}{\sigma_{\theta}^{2}}+\frac{2 \pi \mathrm{SNR}}{h}\left(N \frac{\cos (2 \pi h N)}{\tan (\pi h)}\right. \\
\left.-\sin (2 \pi h N)\left(\frac{1}{2 \sin (\pi h)}+N\right)\right) .
\end{aligned}
$$

4) Weiss-Weinstein Bound: We have to calculate (139)

$$
\begin{aligned}
\eta(\alpha, \beta)= & \ln \int_{\Theta} \frac{p^{\alpha}(\theta+\beta)}{p^{\alpha-1}(\theta)} e^{\frac{\alpha(\alpha-1)}{\sigma^{2}}\|\mathbf{m}(\theta+\beta)-\mathbf{m}(\theta)\|^{2}} d \theta \\
= & \ln \left(e^{2 \alpha(\alpha-1) \operatorname{SNR}\left(N-\sin ^{2}(\pi \beta N)-\frac{1}{2} \frac{\sin (2 \pi \beta N)}{\tan (\pi \beta)}\right)}\right. \\
& \left.\times \int_{\Theta} \frac{p^{\alpha}(\theta+\beta)}{p^{\alpha-1}(\theta)} d \theta\right)
\end{aligned}
$$

thanks to (144) and to the independence of $\theta$ in the term $\| \mathbf{m}(\theta+$ $\beta)-\mathbf{m}(\theta) \|^{2}$.

The remaining term is given by

$$
\begin{aligned}
\int_{\Theta} & \frac{p^{\alpha}(\theta+\beta)}{p^{\alpha-1}(\theta)} d \theta \\
& =\frac{1}{\sqrt{2 \pi} \sigma_{\theta}} e^{-\frac{1}{2 \sigma_{\theta}^{2}}\left[\alpha \beta^{2}-2 \alpha \beta \mu+\mu^{2}\right]} \int_{\Theta} e^{-\frac{1}{2 \sigma_{\theta}^{2}}\left[\theta^{2}+2 \theta(\alpha \beta-\mu)\right]} d \theta \\
& =e^{-\frac{1}{2 \sigma_{\theta}^{2}}\left[\alpha \beta^{2}-2 \alpha \beta \mu+\mu^{2}\right]+\frac{(\alpha \beta-\mu)^{2}}{2 \sigma_{\theta}^{2}}} \\
& =e^{-\frac{\alpha \beta^{2}}{2 \sigma_{\theta}^{2}}(1-\alpha)}
\end{aligned}
$$

where $\int_{\Theta} e^{-\left(1 / 2 \sigma_{\theta}^{2}\right)\left[\theta^{2}+2 \theta(\alpha \beta-m)\right]} d \theta$ is obtained thanks to [71, p. 355 , eq. $(\mathrm{BI}(28)(1))]$.

Consequently, $\eta(\alpha, \beta)$ is given by

$$
\begin{aligned}
\eta(\alpha, \beta)= & \alpha(\alpha-1)(2 \operatorname{SNR}(N \\
& \left.\left.-\sin ^{2}(\pi \beta N)-\frac{1}{2} \frac{\sin (2 \pi \beta N)}{\tan (\pi \beta)}\right)-\frac{\beta^{2}}{2 \sigma_{\theta}^{2}}\right) .
\end{aligned}
$$

\section{ACKNOWLEDGMENT}

This paper was developed while A. Renaux was a Postdoctoral Research Associate in Prof. Nehorai's research group at the Department of Electrical and Systems Engineering, Washington University, St. Louis. 


\section{REFERENCES}

[1] A. Renaux, P. Forster, and P. Larzabal, "A new derivation of the Bayesian bounds for parameter estimation," in Proc. IEEE Statist. Signal Process. Workshop—SSP05, Bordeaux, France, Jul. 2005, pp. $567-572$.

[2] A. Renaux, P. Forster, P. Larzabal, and C. Richmond, "The Bayesian Abel bound on the mean square error," in Proc. IEEE Int. Conf. Acoust., Speech, Signal Process., Toulouse, France, May 2006, vol. 3, pp. 9-12.

[3] R. A. Fisher, "On the mathematical foundations of theoretical statistics," Phil. Trans. Royal Soc., vol. 222, p. 309, 1922.

[4] D. Dugué, "Application des propriétés de la limite au sens du calcul des probabilités à I'étude des diverses questions d'estimation," Ecol. Poly., vol. 3, pp. 305-372, 1937.

[5] M. Frechet, "Sur I'extension de certaines evaluations statistiques an cas de petit echantillons," Rev. Inst. Int. Statist., vol. 11, pp. 182-205, 1943.

[6] G. Darmois, "Sur les lois limites de la dispersion de certaines estimations," Rev. Inst. Int. Statist., vol. 13, pp. 9-15, 1945.

[7] C. R. Rao, "Information and accuracy attainable in the estimation of statistical parameters," Bull. Calcutta Math. Soc., vol. 37, pp. 81-91, 1945.

[8] H. Cramér, Mathematical Methods of Statistics. New York: Princeton Univ. Press, 1946.

[9] L. L. Scharf and T. McWhorter, "Geometry of the Cramer Rao bound," Signal Process., vol. 31, pp. 301-311, 1993.

[10] J. Xavier and V. Barroso, "The Riemannian geometry of certain parameter estimation problems with singular Fisher information matrices," in Proc. IEEE Int. Conf. Acoust., Speech, Signal Process., Montreal, Canada, May 2004, vol. 2, pp. 1021-1024.

[11] J. Xavier and V. Barroso, "Intrinsic variance lower bound (IVLB): An extension of the Cramer Rao bound to Riemannian manifolds," in Proc. IEEE Int. Conf. Acoust., Speech, Signal Process., Hong Kong, Mar. 2004, vol. 5, pp. 1033-1036.

[12] A. Manikas, Differential Geometry in Array Processing. London, U.K.: Imperial College Press, 2004.

[13] S. T. Smith, "Statistical resolution limits and the complexified Cramer Rao bound," IEEE Trans. Signal Process., vol. 53, pp. 1597-1609, May 2005.

[14] S. T. Smith, "Covariance, subspace, and intrinsic Cramer Rao bounds," IEEE Trans. Signal Process., vol. 53, pp. 1610-1630, May 2005.

[15] J.-P. Delmas and H. Abeida, "Stochastic Cramer-Rao bound for noncircular signals with application to DOA estimation," IEEE Trans. Signal Process., vol. 52, pp. 3192-3199, Nov. 2004.

[16] J.-P. Delmas and H. Abeida, "Cramer Rao bounds of DOA estimates for BPSK and QPSK modulated signals," IEEE Trans. Signal Process., vol. 54, pp. 117-126, Jan. 2005.

[17] E. Chaumette, P. Larzabal, and P. Forster, "On the influence of a detection step on lower bounds for deterministic parameter estimation," IEEE Trans. Signal Process., vol. 53, pp. 4080-4090, Nov. 2005.

[18] Q. Zou, Z. Lin, and R. J. Ober, "The Cramer Rao lower bound for bilinear systems," IEEE Trans. Signal Process., vol. 54, pp. 1666-1680, May 2006.

[19] I. Yetik and A. Nehorai, "Performance bounds on image registration," IEEE Trans. Signal Process., vol. 54, pp. 1737-1736, May 2006.

[20] H. L. Van Trees, Detection, Estimation and Modulation Theory. New York: Wiley, 1968, vol. 1.

[21] D. C. Rife and R. R. Boorstyn, "Single tone parameter estimation from discrete time observations," IEEE Trans. Inf. Theory, vol. 20, pp. 591-598, 1974.

[22] A. Bhattacharyya, "On some analogues of the amount of information and their use in statistical estimation," Sankhya Indian J. Statist., vol. 8, pp. 1-14, 201-218, 315-328, 1946.

[23] D. A. S. Fraser and I. Guttman, "Bhattacharyya bounds without regularity assumptions," Ann. Math. Stat., vol. 23, pp. 629-632, 1952.

[24] D. G. Chapman and H. Robbins, "Minimum variance estimation without regularity assumptions," Ann. Math. Stat., vol. 22, pp. 581-586, 1951.

[25] J. Kiefer, "On minimum variance estimators," Ann. Math. Stat., vol. 23, pp. 627-629, 1952.

[26] J. M. Hammersley, "On estimating restricted parametrers," J. Royal Soc. Ser. B, vol. 12, pp. 192-240, 1950.

[27] E. W. Barankin, "Locally best unbiased estimates," Ann. Math. Stat., vol. 20, pp. 477-501, 1949.

[28] R. J. McAulay and L. P. Seidman, "A useful form of the Barankin lower bound and its application to ppm threshold analysis," IEEE Trans. Inf. Theory, vol. 15, pp. 273-279, Mar. 1969.
[29] J. S. Abel, "A bound on mean square estimate error," in Proc. IEEE Int. Conf. Acoust., Speech, Signal Process., Albuquerque, NM, 1990, vol. 3, pp. 1345-1348.

[30] J. S. Abel, "A bound on mean square estimate error," IEEE Trans. Inf. Theory, vol. 39, pp. 1675-1680, Sep. 1993.

[31] R. J. McAulay and E. M. Hofstetter, "Barankin bounds on parameter estimation," IEEE Trans. Inf. Theory, vol. 17, pp. 669-676, Nov. 1971.

[32] A. Quinlan, E. Chaumette, and P. Larzabal, "A direct method to generate approximations of the Barankin bound," in Proc. IEEE Int. Conf. Acoust., Speech, Signal Process., Toulouse, France, May 2006, vol. 3, pp. 808-811.

[33] J. Ziv and M. Zakai, "Some lower bounds on signal parameter estimation," IEEE Trans. Inf. Theory, vol. 15, pp. 386-391, May 1969.

[34] S. Bellini and G. Tartara, "Bounds on error in signal parameter estimation," IEEE Trans. Commun., vol. 22, pp. 340-342, Mar. 1974.

[35] D. Chazan, M. Zakai, and J. Ziv, "Improved lower bounds on signal parameter estimation," IEEE Trans. Inf. Theory, vol. 21, pp. 90-93, Jan. 1975.

[36] E. Weinstein, "Relations between Belini-Tartara, Chazan-Zakai-Ziv, and Wax-Ziv lower bounds," IEEE Trans. Inf. Theory, vol. 34, pp. 342-343, Mar. 1988.

[37] K. L. Bell, Y. Steinberg, Y. Ephraim, and H. L. V. Trees, "Extended Ziv Zakai lower bound for vector parameter estimation," IEEE Trans. Signal Process., vol. 43, pp. 624-638, Mar. 1997.

[38] K. L. Bell, "Performance bounds in parameter estimation with application to bearing estimation," Ph.D. dissertation, George Mason Univ., Fairfax, VA, 1995.

[39] B. Z. Bobrovsky, E. Mayer-Wolf, and M. Zakai, "Some classes of global Cramer Rao bounds," Ann. Statist., vol. 15, pp. 1421-1438, 1987

[40] B. Z. Bobrovsky and M. Zakai, "A lower bound on the estimation error for certain diffusion processes," IEEE Trans. Inf. Theory, vol. 22, pp. 45-52, Jan. 1976.

[41] I. Reuven and H. Messer, "A Barankin-type lower bound on the estimation error of a hybrid parameter vector," IEEE Trans. Inf. Theory, vol. 43, no. 3, pp. 1084-1093, May 1997.

[42] A. J. Weiss and E. Weinstein, "A lower bound on the mean square error in random parameter estimation," IEEE Trans. Inf. Theory, vol. 31, pp. 680-682, Sep. 1985.

[43] , H. L. Van Trees and K. L. Bell, Eds., Bayesian Bounds for Parameter Estimation and Nonlinear Filtering/Tracking. New York: Wiley, 2007.

[44] A. B. Baggeroer, Barankin Bound on the Variance of Estimates of Gaussian Random Process MIT Lincoln Lab., Lexington, MA, Tech. Rep., Jan. 1969.

[45] I. Reuven and H. Messer, "The use of the Barankin bound for determining the threshold SNR in estimating the bearing of a source in the presence of another," in Proc. IEEE Int. Conf. Acoust., Speech, Signal Process., Detroit, MI, May 1995, vol. 3, pp. 1645-1648.

[46] L. Knockaert, "The Barankin bound and threshold behavior in frequency estimation," IEEE Trans. Signal Process., vol. 45, pp. 2398-2401, Sep. 1997.

[47] J. Tabrikian and J. L. Krolik, "Barankin bound for source localization in shallow water," in Proc. IEEE Int. Conf. Acoust., Speech, Signal Process., Munich, Germany, Apr. 1997.

[48] T. L. Marzetta, "Computing the Barankin bound by solving an unconstrained quadratic optimization problem," in Proc. IEEE Int. Conf. Acoust., Speech, Signal Process., Munich, Germany, Apr. 1997, vol. 5, pp. 3829-3832.

[49] I. Reuven and H. Messer, "On the effect of nuisance parameters on the threshold SNR value of the Barankin bound," IEEE Trans. Signal Process., vol. 47, no. 2, pp. 523-527, Feb. 1999.

[50] J. Tabrikian and J. Krolik, "Barankin bounds for source localization in an uncertain ocean environment," IEEE Trans. Signal Process., vol. 47, pp. 2917-2927, Nov. 1999.

[51] A. Ferrari and J.-Y. Tourneret, "Barankin lower bound for change points in independent sequences," in Proc. IEEE Statist. Signal Process. Workshop-SSP03, St. Louis, MO, Sep. 2003, pp. 557-560.

[52] P. Ciblat, M. Ghogho, P. Forster, and P. Larzabal, "Harmonic retrieval in the presence of non-circular Gaussian multiplicative noise: Performance bounds," Signal Process., vol. 85, pp. 737-749, Apr. 2005.

[53] P. Ciblat, P. Forster, and P. Larzabal, "Harmonic retrieval in noncircular complex-valued multiplicative noise: Barankin bound," in Proc. EUS1PCO, Vienne, Australia, Sep. 2004, pp. 2151-2154.

[54] L. Atallah, J. P. Barbot, and P. Larzabal, "SNR threshold indicator in data aided frequency synchronization," IEEE Signal Process. Lett., vol. 11, pp. 652-654, Aug. 2004. 
[55] L. Atallah, J. P. Barbot, and P. Larzabal, "From Chapman Robbins bound towards Barankin bound in threshold behavior prediction," Electron. Lett., vol. 40, pp. 279-280, Feb. 2004.

[56] A. Renaux, L. N. Atallah, P. Forster, and P. Larzabal, "A useful form of the Abel bound and its application to estimator threshold prediction," IEEE Trans. Signal Process., vol. 55, no. 5, pp. 2365-2369, May 2007.

[57] W. Xu, "Performances bounds on matched-field methods for source localization and estimation of ocean environmental parameters," Ph.D. dissertation, Mass. Inst. Technol., Cambridge, Jun. 2001.

[58] W. Xu, A. B. Baggeroer, and K. Bell, "A bound on mean-square estimation with background parameter mismatch," IEEE Trans. Inf. Theory, vol. 50, pp. 621-632, Apr. 2004.

[59] W. Xu, A. B. Baggeroer, and C. D. Richmond, "Bayesian bounds for matched-field parameter estimation," IEEE Trans. Signal Process., vol. 52, pp. 3293-3305, Dec. 2004.

[60] A. J. Weiss and E. Weinstein, "Fundamental limitation in passive time delay estimation Part 1: Narrowband systems," IEEE Trans. Acoust., Speech, Signal Process., vol. 31, pp. 472-486, Apr. 1983.

[61] K. L. Bell, Y. Ephraim, and H. L. V. Trees, "Ziv Zakai lower bounds in bearing estimation," in Proc. IEEE Int. Conf. Acoust., Speech, Signal Process., Detroit, MI, 1995, vol. 5, pp. 2852-2855.

[62] K. L. Bell, Y. Ephraim, and H. L. V. Trees, "Explicit Ziv Zakai lower bound for bearing estimation," IEEE Trans. Signal Process., vol. 44, pp. 2810-2824, Nov. 1996.

[63] P. Ciblat and M. Ghogho, "Ziv Zakai bound for harmonic retrieval in multiplicative and additive Gaussian noise," in Proc. IEEE Statist. Signal Process. Workshop—SSP05, Bordeaux, France, Jul. 2005, pp. $561-566$.

[64] A. Renaux, "Weiss-Weinstein bound for data aided carrier estimation," IEEE Signal Process. Lett., vol. 14, no. 4, pp. 283-286, Apr. 2007.

[65] F. E. Glave, "A new look at the Barankin lower bound," IEEE Trans. Inf. Theory, vol. 18, no. 3, pp. 349-356, May 1972.

[66] P. Forster and P. Larzabal, "On lower bounds for deterministic parameter estimation," in Proc. IEEE Int. Conf. Acoust., Speech, Signal Process., Orlando, FL, 2002, vol. 2, pp. 1137-1140.

[67] E. Weinstein and A. J. Weiss, "A general class of lower bounds in parameter estimation," IEEE Trans. Inf. Theory, vol. 34, pp. 338-342, Mar. 1988.

[68] S. M. Kay, Fundamentals of Statistical Signal Processing. Englewood Cliffs, NJ: Prentice-Hall, 1993, vol. I.

[69] A. Renaux, "Contribution a panalyse des performances d'estimaiion en traitement statisticue du signal" Ph.D. dissertation, Ecole Normale Superieure de Cachan, Cachan, France, Jul. 2006 [Online]. Available: http://www.satie.ens-c;achan.fr/ts/These_Alex.pdf

[70] H. L. Van Trees, Detection, Estimation and Modulation Theory: RadarSonar Signal Processing and Gaussian Signals in Noise. New York: Wiley, 2001, vol. 3.

[71] S. Gradshteyn and I. M. Ryzhik, Table of Integrals, Series, and Products. San Diego, CA: Academic, 1994.

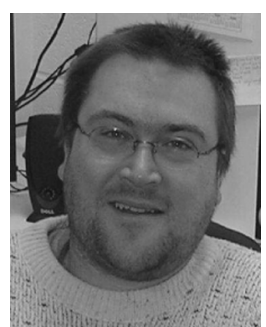

Alexandre Renaux (S'06-M'08) comes from a small French mountain called "les Vosges." $\mathrm{He}$ received the Agrégation, the M.Sc., and the Ph.D. degrees in electrical engineering from the École Normale Supérieure de Cachan, France, in 2002, 2003, and 2006, respectively.

From 2006 to 2007, he was a Postdoctoral Research Associate with the Department of Electrical and Systems Engineering, Washington University, St. Louis, MO. He is currently an Assistant Professor with the Department of Physics and a Member of the Laboratory of Signals and Systems (L2S), University Paris 11, France. His research interests include detection and parameter estimation theory in statistical signal and array processing.

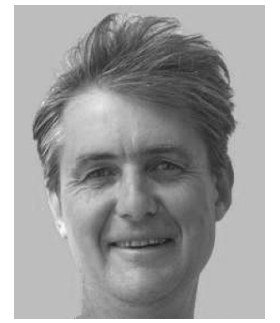

Philippe Forster (M'89) was born in Brest, France, in 1960. He received the Agrégation de Physique Appliquée degree from the Ecole Normale Supérieure de Cachan, France, in 1983, and the Ph.D. degree in electrical engineering in 1988 from the University of Rennes, France.

$\mathrm{He}$ is currently a Professor of Electrical Engineering with the Université de Nanterre, IUT de Ville d'Avray, France, and member of the SATIE Laboratory, École Normale Supérieure de Cachan, France. His research interests are in the field of statistical signal processing and array processing.

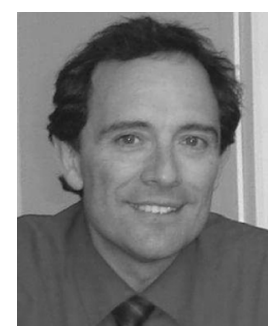

Pascal Larzabal (M'93) was born in the Basque country in the south of France in 1962. He received the Agrégation in Electrical Engineering in 1988, the $\mathrm{Ph} . \mathrm{D}$. degree in 1992, and the "habilitation à diriger les recherches" in 1998, all from the École Normale Supérieure of Cachan, France.

$\mathrm{He}$ is now Professor of Electrical Engineering with the University of Paris-Sud 11, France. He teaches electronics, signal processing, control, and mathematics. From 1998 to 2003, he was the Director of IUP GEII, University of Paris-Sud 11. From March 2003 to March 2007, he was Head of the Electrical Engineering Department, Institut Universitaire de Technologie of Cachan. Since January 2007, he has been the Director of the Laboratory SATIE (UMR CNRS 8029 École Normale Supérieure de Cachan). Since 1993, he has been Head of the Signal Processing Group, Laboratory SATIE. His research concerns estimation in array processing and spectral analysis for wave-front identification, radars, communications, tomography, and medical imaging. His recent works concern estimator performances and associated minimal bounds.

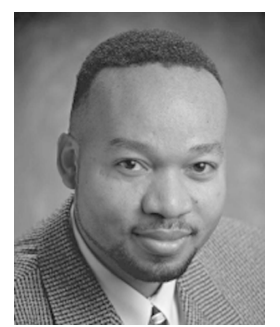

Christ D. Richmond (M'99-SM'05) received the B.S. degree in electrical engineering from the University of Maryland, College Park, and the B.S. degree in mathematics from Bowie State University, Bowie, MD. He received the S.M., E.E., and Ph.D. degrees in electrical engineering from the Massachusetts Institute of Technology (MIT), Cambridge.

$\mathrm{He}$ is currently a member of the Technical Research Staff at MIT Lincoln Laboratory, Lexington. His research interests include detection and parameter estimation theory, sensor array and multichannel signal processing, statistical signal processing, random matrix theory, radar/sonar signal processing, multivariate statistical analysis, information theory, multiuser detection, multiinput/multioutput (MIMO) systems, and wireless communications.

Dr. Richmond is the recipient of the Office of Naval Research Graduate Fellowship Award 1990-1994, the Alan Berman Research Publications Award March 1994 (Naval Research Laboratory), and the IEEE Signal Processing Society 1999 Young Author Best Paper Award in area of Sensor Array and Multi-channel (SAM) Signal Processing. He served as the Technical Chairman of the Adaptive Sensor Array Processing (ASAP) Workshop, MIT Lincoln Laboratory, 2007, 2006, and 1998, and served as a member the IEEE Technical Committee on SAM Signal Processing. He was an Associate Editor for the IEEE TRANSACTIONS ON SignAl Processing from 2002 to 2005. He was an invited reviewer for the book Bayesian Bounds for Parameter Estimation and Nonlinear Filtering/Tracking, by Prof. H. L. Van Trees and Prof. K. Bell of George Mason University, Eds. (Piscataway, NJ: IEEE, 2007). 


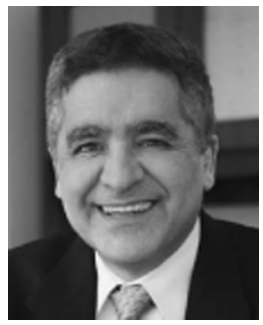

Arye Nehorai (S'80-M'83-SM'90-F'94) received the B.Sc. and M.Sc. degrees in electrical engineering from the Technion, Israel, and the Ph.D. degree in electrical engineering from Stanford University, Stanford, CA.

From 1985 to 1995 , he was a faculty member with the Department of Electrical Engineering, Yale University, New Haven, CT. In 1995, he joined the Department of Electrical Engineering and Computer Science, The University of Illinois at Chicago (UIC) as Full Professor. From 2000 to 2001, he was Chair of the department's Electrical and Computer Engineering (ECE) Division, which then became a new department. In 2001, he was named University Scholar of the University of Illinois. In 2006, he became Chairman of the Department of Electrical and Systems Engineering, Washington University, St. Louis (WUSTL), MO. He is the inaugural holder of the Eugene and Martha
Lohman Professorship and the Director of the Center for Sensor Signal and Information Processing (CSSIP) at WUSTL since 2006.

Dr. Nehorai was Editor-in-Chief of the IEEE TRANSACTIONS ON SIGNAL PROCESSING during 2000 to 2002. During 2003-2005, he was Vice President (Publications) of the IEEE Signal Processing Society, Chair of the Publications Board, member of the Board of Governors, and member of the Executive Committee of this Society. From 2003 to 2006, he was the Founding Editor of the Special Columns on Leadership Reflections in the IEEE SIGNAL PROCESSING MAGAZINE. He was corecipient of the IEEE SPS 1989 Senior Award for Best Paper with P. Stoica, coauthor of the 2003 Young Author Best Paper Award, and corecipient of the 2004 Magazine Paper Award with A. Dogandzic. He was elected Distinguished Lecturer of the IEEE SPS for the term 2004 to 2005 and received the 2006 IEEE SPS Technical Achievement Award. He is the Principal Investigator of the new multidisciplinary university research initiative (MURI) project entitled Adaptive Waveform Diversity for Full Spectral Dominance. He has been a Fellow of the Royal Statistical Society since 1996. 